Pacific

Journal of

Mathematics

CAPITULATION DES 2-CLASSES D'IDÉAUX DE CERTAINS CORPS BIQUADRATIQUES DONT LE CORPS DE GENRES DIFFÈRE DU 2-CORPS DE CLASSES DE HILBERT

Abdelmalek Azizi et Ali Mouhib 


\title{
CAPITULATION DES 2-CLASSES D'IDÉAUX DE CERTAINS CORPS BIQUADRATIQUES DONT LE CORPS DE GENRES DIFFÈRE DU 2-CORPS DE CLASSES DE HILBERT
}

\author{
Abdelmalek Azizi et Ali MouhiB
}

Let $K=\mathbb{Q}\left(\sqrt{d_{1}}, \sqrt{d_{2}}\right)$, where $d_{1}$ and $d_{2}$ are positive square-free integers such that $\left(d_{1}, d_{2}\right)=1$. Let $K_{2}^{(1)}$ be the Hilbert 2-class field of $K$. Let $K_{2}^{(2)}$ be the Hilbert 2-class field of $K_{2}^{(1)}$ and $K^{(*)}$ the genus field of $K$. We suppose that $K_{2}^{(1)} \neq K^{(*)}$ and $\operatorname{Gal}\left(K_{2}^{(1)} / K\right) \simeq \mathbb{Z} / 2 \mathbb{Z} \times \mathbb{Z} / 2 \mathbb{Z}$. We study the capitulation problem of the 2-ideal classes of $K$ in the sub-extensions of $K_{2}^{(1)} / K$ and we determine the structure of $\operatorname{Gal}\left(K_{2}^{(2)}\right)$.

\section{Introduction}

Soient $K$ un corps de nombres, $K_{2}^{(1)}$ le 2-corps de classes de Hilbert de $K, K_{2}^{(2)}$ le 2-corps de classes de Hilbert de $K_{2}^{(1)}, G$ le groupe $\operatorname{Gal}\left(K_{2}^{(2)} / K\right)$ et $K^{(*)}$ le corps de genres de $K$, c'est-à-dire la plus grande extension de $K$ da la forme $K L$ non ramifiée pour tous les premiers finis et infinis et telle que $L / \mathbb{Q}$ est abélienne.

On suppose que $\operatorname{Gal}\left(K_{2}^{(1)} / K\right) \simeq \mathbb{Z} / 2 \mathbb{Z} \times \mathbb{Z} / 2 \mathbb{Z}$; alors $K_{2}^{(1)}$ contient trois sousextensions quadratiques sur $K$ qu'on désigne par $K_{1}, K_{2}$ et $K_{3}$. Kisilevsky [1976] a lié le problème de capitulation dans les extensions $K_{1} / K, K_{2} / K$ et $K_{3} / K$ à la structure du groupe $G$ et aussi à la structure de la 2-partie du groupe de classes de ces trois extensions. Plus précisément, Olga Taussky a démontré que le groupe $G$ est abélien, diédral, quaternionique ou semi-diédral (voir par exemple [Gorenstein 1980]). Par conséquent, il existe $i_{0} \in\{1,2,3\}$ tel que le 2-groupe de classes de $K_{i_{0}}$ est cyclique et on a deux possibilités:

1) Les 2-groupes de classes des extensions $K_{1}, K_{2}$ et $K_{3}$ sont cycliques et dans ce cas $G$ est abélien ou quaternionique d'ordre 8 . Plus précisément:

- $G$ est abélien si et seulement si pour tout $i \in\{1,2,3\}$ toutes les 2 -classes de $K$ capitulent dans $K_{i}$.

- $G$ est quaternionique d'ordre 8 si et seulement si pour tout $i \in\{1,2,3\}$, il existe une seule 2-classe non triviale de $K$ qui capitule dans $K_{i}$.

2) Le 2-groupe de classes de $K_{i_{0}}$ est cyclique et pour $i \neq i_{0}$, le 2-groupe de classes de $K_{i}$ est de type $(2,2)$ et dans ce cas: 
- Pour $i \neq i_{0}$, il existe une seule 2-classe non triviale de $K$ qui capitule dans $K_{i}$.

- $G$ est diédral si et seulement si toutes les 2-classes de $K$ capitulent dans $K_{i_{0}}$.

- $G$ est quaternionique d'ordre superieur ou égal à $2^{4}$ si et seulement si il existe une seule 2-classe non triviale de $K$ qui capitule dans $K_{i_{0}}$ et $K_{i_{0}}$ est de type (A). (Pour la définition du type (A) et du type (B), voir par exemple [Kisilevsky 1976].)

- $G$ est semi-diédral d'ordre superieur ou égal à $2^{4}$ si et seulement si il existe une seule 2-classe non triviale de $K$ qui capitule dans $K_{i_{0}}$ et $K_{i_{0}}$ est de type (B).

Ces résultats nous permettent de réduire l'étude du problème de capitulation dans les extensions intermédiaires de $K_{2}^{(1)} / K$ à une étude dans $K_{i_{0}}$.

Si $K$ est un corps biquadratique et $K_{2}^{(1)} \neq K^{(*)}$, alors d'après la théorie des groupes $K^{(*)} \neq K$ (car sinon $\operatorname{Gal}\left(K_{2}^{(1)} / K\right)$ serait cyclique) et par suite $K^{(*)}$ est l'un des corps $K_{1}, K_{2}$ ou $K_{3}$.

Dans [Azizi et Mouhib $\geq 2008$ ], on a démontré que si $K_{2}^{(1)} / K$ a $\mathbb{Z} / 2 \mathbb{Z} \times \mathbb{Z} / 2 \mathbb{Z}$ pour Galois groupe et $\left[K_{2}^{(1)}: K^{(*)}\right]=2$, alors:

1) Le 2-groupe de classes de $K^{(*)}$ est cyclique et $K_{2}^{(2)}$ est le 2-corps de classes de Hilbert de $K^{(*)}$.

2) Le groupe $G$ ne peut jamais être semi-diédral.

Dans ce travail, on suppose que $K=\mathbb{Q}\left(\sqrt{d_{1}}, \sqrt{d_{2}}\right)$ où $d_{1}$ et $d_{2}$ sont deux entiers naturels sans facteurs carrés et premiers entre eux, $\operatorname{Gal}\left(K_{2}^{(1)} / K\right) \simeq \mathbb{Z} / 2 \mathbb{Z} \times \mathbb{Z} / 2 \mathbb{Z}$ et $K_{2}^{(1)} \neq K^{(*)}$. Alors on étudie le problème de capitulation des 2-classes d'idéaux de $K$ en distinguant deux cas:

1) L'extension $K / \mathbb{Q}\left(\sqrt{d_{1} d_{2}}\right)$ est non ramifiée.

2) L'extension $K / \mathbb{Q}\left(\sqrt{d_{1} d_{2}}\right)$ est ramifiée.

Avant d'étudier ces cas, on aura besoin de certains résultats sur le rang du 2groupe de classes des corps biquadratiques réels contenant un corps quadratique de nombre de classes impair. Dans toute la suite, on désigne par $h(n)$ la 2-partie du nombre de classes de $\mathbb{Q}(\sqrt{n})$ et par $h(M)$ la 2-partie du nombre de classes d'un corps quelconque $M$.

\section{Rang du 2-groupe de classes de certains corps biquadratiques réels}

Dans ce paragraphe on suppose que $K=\mathbb{Q}(\sqrt{m}, \sqrt{d})$ où $m$ et $d$ sont deux entiers naturels sans facteurs carrés et le nombre de classes de $\mathbb{Q}(\sqrt{m})$ est impair. On désigne par $\varepsilon_{m}$ l'unité fondamentale de $\mathbb{Q}(\sqrt{m}), E$ le groupe des unités de $\mathbb{Q}(\sqrt{m})$, $r$ le nombre des premiers de $\mathbb{Q}(\sqrt{m})$ ramifiés dans $K, \mathcal{N}$ l'application norme par rapport à l'extension $K / \mathbb{Q}(\sqrt{m})$, e l'entier naturel défini par $2^{e}=\left[E: E \cap \mathcal{N}(K)^{*}\right]$ et par $C_{2, K}$ le 2-groupe de classes de $K$. Dans [Azizi et Mouhib 2001], on a 
montré que le rang de $C_{2, K}$ est $r-1-e$. La détermination de l'entier $e$ revient à chercher quand est-ce que les éléments $-1, \varepsilon_{m}$ et $-\varepsilon_{m}$ sont normes ou non dans l'extension $K / \mathbb{Q}(\sqrt{m})$. Ce qui revient à chercher les valeurs du symbole du reste normique $\left(\frac{-1, d}{\rho \mathcal{P}}\right),\left(\frac{\varepsilon_{m}, d}{\rho^{p}}\right)$ pour tous les premiers de $\mathbb{Q}(\sqrt{m})$ ramifiés dans $K$. Ainsi $e \in\{0,1,2\}$; plus précisément:

$e=0$ si et seulement si -1 et $\varepsilon_{m}$ sont des normes dans l'extension $K / \mathbb{Q}(\sqrt{m})$.

$e=2$ si et seulement si $-1, \varepsilon_{m}$ et $-\varepsilon_{m}$ ne sont pas des normes dans l'extension $K / \mathbb{Q}(\sqrt{m})$. D'autre part, d'après la théorie des genres $m$ est de l'une des formes suivantes:

1) $m$ est un premier.

2) $m=2 q$ où $q$ est un premier tel que $q \equiv-1(\bmod 4)$.

3) $m=q^{\prime} q^{\prime \prime}$ où $q^{\prime}$ et $q^{\prime \prime}$ sont deux premiers tels que $q^{\prime} \equiv q^{\prime \prime} \equiv-1(\bmod 4)$.

Théorème 1 [Azizi et Mouhib 2001]. Soient $m$ un premier $\equiv 1$ ou $2(\bmod 4)$ et $K=\mathbb{Q}(\sqrt{m}, \sqrt{d})$ où d est un entier naturel sans facteurs carrés. Posons

$$
S=\left\{q_{1} \text { divisant } d \mid\left(\frac{m}{q_{1}}\right)=1 \text { et } q_{1} \text { premier impair de } \mathbb{Q}\right\} .
$$

S'il existe un premier $q$ tel que $q \equiv 3(\bmod 4)$ et $q \mid d$, alors $e=1$ ou $e=2$. Plus précisément :

1) Si $m \equiv 2$ ou $5(\bmod 8)$, alors $\operatorname{rang}\left(C_{2, K}\right)=r-2$ si et seulement si $\left(\frac{-1}{q_{1}}\right)=1$ pour tout $q_{1} \in S$.

2) Si $m \equiv 1(\bmod 8)$, alors rang $\left(C_{2, K}\right)=r-2$ si et seulement si $\left(\frac{-1}{q_{1}}\right)=1$ pour tout $q_{1} \in S$ et

$$
\left[d=2 \text { c avec }\left(\frac{-1}{c}\right)=1\right] \text { ou bien } d \equiv 1(\bmod 4) .
$$

Théorème 2 [Azizi et Mouhib 2001]. Soient $m$ un premier $\equiv 1$ ou $2(\bmod 4)$ et $K=\mathbb{Q}(\sqrt{m}, \sqrt{d})$ où $d$ est un entier naturel sans facteurs carrés, qui n'est pas divisible par les premiers $\equiv-1(\bmod 4)$. Alors $e=0$ ou $e=1$; plus précisément :

1) Si $m=p \equiv 1(\bmod 4)$, alors $\operatorname{rang}\left(C_{2, K}\right)=r-1$ si et seulement si pour chaque $q \mid d$ tel que $\left(\frac{q}{p}\right)=1$ on $a\left(\frac{q}{p}\right)_{4}=\left(\frac{p}{q}\right)_{4}$ et

$$
\left(\frac{2}{p}\right)_{4}=(-1)^{(p-1) / 8} \text { si } p \equiv 1(\bmod 8) \text { et } d=2 c \text {. }
$$

2) Si $m=2$, alors rang $\left(C_{2, K}\right)=r-1$ si et seulement si pour chaque $q \mid d$ tel que $q \equiv 1(\bmod 8)$ on $a\left(\frac{2}{q}\right)_{4}=(-1)^{(q-1) / 8}$.

Dans le cas où $m \in\left\{q, 2 q, q^{\prime} q^{\prime \prime}\right\}$ où $q, q^{\prime}$ et $q^{\prime \prime}$ sont des premiers tels que $q \equiv q^{\prime} \equiv q^{\prime \prime} \equiv-1(\bmod 4)$, on vérifie facilement que l'unité fondamentale $\varepsilon_{m}$ de $\mathbb{Q}(\sqrt{m})$ vérifie $\varepsilon_{m}=a_{m} u^{2}$ où $u \in \mathbb{Q}(\sqrt{m})$ et $a_{m} \in \mathbb{N}$ est défini comme suit: $a_{m}=2$ si $m=q$ ou $m=2 q$ et $a_{m}=q^{\prime}$ ou $a_{m}=q^{\prime \prime}$ si $m=q^{\prime} q^{\prime \prime}$. Alors en utilisant les propriétés du symbole du reste normique (voir par exemple [Hasse 1930]), on démontre les deux théorèmes suivants: 
Théorème 3. Soient $q, q^{\prime}$ et $q^{\prime \prime}$ des premiers tels que $q \equiv q^{\prime} \equiv q^{\prime \prime} \equiv-1(\bmod 4), m$ un entier naturel tel que $m \in\{q, 2 q\}$ ou $m=q^{\prime} q^{\prime \prime} \equiv 5(\bmod 8)$ et $K=\mathbb{Q}(\sqrt{m}, \sqrt{d})$ où $d$ est un entier naturel sans facteurs carrés. On pose

$$
S=\left\{q_{1} \text { divisant } d \mid\left(\frac{m}{q_{1}}\right)=1 \text { et } q_{1} \text { premier impair de } \mathbb{Q}\right\} .
$$

Alors:

$e=0$ si et seulement si $\left(\frac{-1}{q_{1}}\right)=\left(\frac{a_{m}}{q_{1}}\right)=1$ pour tout $q_{1} \in S$.

$e=2$ si et seulement si il existe des premiers $q_{1}, q_{2}, q_{3}$ de $S$ tels que $\left(\frac{-1}{q_{1}}\right)=$ $\left(\frac{a_{m}}{q_{1}}\right)=-1$ et $\left(\frac{-1}{q_{3}}\right) \neq\left(\frac{a_{m}}{q_{3}}\right)$.

Preuve. On suppose que $m \in\left\{q^{\prime}, 2 q^{\prime}\right\}$ ou $m=q^{\prime} q^{\prime \prime} \equiv 5(\bmod 8)$. Soit $\mathscr{P}$ un idéal premier de $\mathbb{Q}(\sqrt{m})$ qui se ramifie dans $K$.

Calculons le symbole du reste normique $\left(\frac{-1, d}{\mathscr{P}}\right)$. Si $\mathscr{P}$ est au dessus d'un premier $q_{1}$ impair tel que $\left(\frac{m}{q_{1}}\right)=-1$, alors d'après les propriétés du symbole du reste normique (voir par exemple [Hasse 1930]), on a

$$
\left(\frac{-1, d}{\mathscr{P}}\right)=\left(\frac{\mathbb{N}_{\mathbb{Q}(\sqrt{m}) / Q}(-1), d}{q_{1}}\right)=1 .
$$

Si $\mathscr{P}$ est au dessus de $q_{1}$ où $q_{1} \in S$, alors $\left(\frac{-1, d}{\mathscr{P}}\right)=\left(\frac{d,-1}{\mathscr{P}}\right)=\left(\frac{-1}{\mathscr{P}}\right)^{v_{\mathcal{P}}(d)}=\left(\frac{-1}{q_{1}}\right)$ où $v_{\mathscr{P}}(d)$ désigne la valuation d'indice $\mathscr{P}$ appliquée à l'idéal engendré par $(d)$ dans $\mathbb{Q}(\sqrt{m})$. Si $\mathscr{P}$ est au dessus de 2 , alors $\mathscr{P}$ est le seul premier de $k$ qui est au dessus de 2. Ainsi, d'après [Hasse 1930], on a

$$
\left(\frac{-1, d}{\mathscr{P}}\right)=\left(\frac{\mathbb{N}_{\mathbb{Q}(\sqrt{m}) / \mathbb{Q}}(-1), d}{2}\right)=1 .
$$

On en déduit alors que -1 est norme dans l'extension $K / \mathbb{Q}(\sqrt{m})$ si et seulement si pour tout premier $q_{1} \in S$, on a $\left(\frac{-1}{q_{1}}\right)=1$.

Calculons le symbole du reste normique $\left(\frac{\varepsilon_{m}, d}{\rho^{\prime}}\right)$. On sait que $\varepsilon_{m}=a_{m} u^{2}$ où $u \in \mathbb{Q}(\sqrt{m})$. Ainsi on a $\left(\frac{\varepsilon_{m}, d}{\mathscr{P}}\right)=\left(\frac{a_{m}, d}{\mathscr{P}}\right)$. Si $\mathscr{P}$ est au dessus de $q_{1}$ où $q_{1}$ est un premier impair tel que $\left(\frac{m}{q_{1}}\right)=-1$, on trouve que

$$
\left(\frac{\varepsilon_{m}, d}{\mathscr{P}}\right)=\left(\frac{\mathcal{N}_{\mathbb{Q}(\sqrt{m} / \mathbb{Q}}\left(\varepsilon_{m}\right), d}{q_{1}}\right)=1 .
$$

Si $\mathscr{P}$ est au dessus de $q_{1}$ où $q_{1} \in S$, alors $\left(\frac{\varepsilon_{m}, d}{\mathscr{P}}\right)=\left(\frac{a_{m}, d}{\mathscr{P}}\right)=\left(\frac{d, a_{m}}{\mathscr{P}}\right)=\left(\frac{a_{m}}{\mathscr{P}}\right)^{v_{\mathscr{P}}}(d)=$ $\left(\frac{a_{m}}{q_{1}}\right)$. Si $\mathscr{P}$ est au dessus de 2 , on trouve que $\left(\frac{\varepsilon_{m}, d}{\mathscr{P}}\right)=1$. Ainsi $\varepsilon_{m}$ est norme dans l'extension $K / \mathbb{Q}(\sqrt{m})$ si et seulement si pour tout $q_{1} \in S$, on a $\left(\frac{a_{m}}{q_{1}}\right)=1$. Ce qui termine la preuve du théorème.

Théorème 4. Soient $q^{\prime}$ et $q^{\prime \prime}$ deux premiers tels que $q^{\prime} \equiv q^{\prime \prime} \equiv-1(\bmod 4), m$ un entier naturel tel que $m=q^{\prime} q^{\prime \prime} \equiv 1(\bmod 8)$ et $K=\mathbb{Q}(\sqrt{m}, \sqrt{d})$ où $d$ est un entier naturel sans facteurs carrés. On pose

$$
S=\left\{q_{1} \text { divisant } d \mid\left(\frac{m}{q_{1}}\right)=1 \text { et } q_{1} \text { premier impair de } \mathbb{Q}\right\} .
$$


Alors $e=0$ si et seulement si $\left(\frac{-1}{q_{1}}\right)=\left(\frac{a_{m}}{q_{1}}\right)=1$ pour tout $q_{1} \in S$ et

$$
d \equiv 1(\bmod 4) \text { ou } d=2 \text { c tel que }\left(\frac{-1}{c}\right)=\left(\frac{2}{q^{\prime}}\right)=1 \text {. }
$$

$e=2$ si et seulement si l'une des conditions suivantes est vérifiée:

- $d \equiv-1(\bmod 4)$ et $\exists q_{1} \in S$ tel que $\left(\frac{-1}{q_{1}}\right) \neq\left(\frac{a_{m}}{q_{1}}\right)$.

- $d \equiv 1$ (mod 4) et $\exists q_{1}, q_{2}, q_{3} \in S$ tels que $\left(\frac{-1}{q_{1}}\right)=\left(\frac{a_{m}}{q_{2}}\right)=-1$ et $\left(\frac{-1}{q_{3}}\right) \neq\left(\frac{a_{m}}{q_{3}}\right)$.

- $d=2 c$ et

$$
\begin{aligned}
& \left(\frac{2}{q^{\prime}}\right)=-\left(\frac{-1}{c}\right)=1 \text { et } \exists q_{1} \in S \text { tel que }\left(\frac{-1}{q_{1}}\right) \neq\left(\frac{a_{m}}{q_{1}}\right) \text { ou } \\
& \left(\frac{-1}{c}\right)=-\left(\frac{2}{q^{\prime}}\right)=1 \text { et } \exists q_{1} \in S \text { tel que }\left(\frac{-1}{q_{1}}\right)=-1 \text { ou } \\
& \left(\frac{-1}{c}\right)=\left(\frac{2}{q^{\prime}}\right)=1 \text { et } \exists q_{1}, q_{2}, q_{3} \in S \text { tels que }\left(\frac{-1}{q_{1}}\right)=\left(\frac{a_{m}}{q_{2}}\right)=-1 \text { et }\left(\frac{-1}{q_{3}}\right) \neq\left(\frac{a_{m}}{q_{3}}\right) .
\end{aligned}
$$

Preuve. On suppose que $m=q^{\prime} q^{\prime \prime} \equiv 1(\bmod 8)$. Soit $\mathscr{P}$ un premier de $\mathbb{Q}(\sqrt{m})$ qui se ramifie dans $K$. Calculons $\left(\frac{-1, d}{\mathscr{P}}\right)$. Si $\mathscr{P}$ est au dessus d'un premier $q_{1}$ impair, alors comme dans le théorème 3 , on trouve que:

Si $\left(\frac{m}{q_{1}}\right)=-1$, on a $\left(\frac{-1, d}{\mathscr{P}}\right)=1$. Si $\left(\frac{m}{q_{1}}\right)=1$, on a $\left(\frac{-1, d}{\mathscr{P}}\right)=\left(\frac{-1}{q_{1}}\right)$. Si $\mathscr{P}$ est au dessus de 2, on raisonne comme suit:

Supposons que $d \equiv 3(\bmod 4)$, alors on a $\left(\frac{-1, q^{\prime} d}{\mathscr{P}}\right)=\left(\frac{-1, q^{\prime}}{\mathscr{P}}\right)\left(\frac{-1, d}{\mathscr{P}}\right)$. Comme $q^{\prime} d \equiv 1(\bmod 4)$, alors $\mathscr{P}$ ne se ramifie pas dans $\mathbb{Q}\left(\sqrt{m}, \sqrt{q^{\prime} d}\right)$; par suite $\left(\frac{-1, q^{\prime} d}{\rho p}\right)=$ $\left(\frac{q^{\prime} d}{\mathscr{P}}\right)_{v_{\mathscr{P}}}(-1)=1$. Ainsi on a $\left(\frac{-1, d}{\mathscr{P}}\right)=\left(\frac{-1, q^{\prime}}{\mathscr{P}}\right)$. On se ramène à calculer le symbole $\left(\frac{-1, q^{\prime}}{\mathscr{P}}\right)$. Soient le corps $L=\mathbb{Q}\left(\sqrt{q^{\prime}}, \sqrt{q^{\prime \prime}}\right)$ et $C_{2, L}$ son 2-groupe de classes. D'après le théorème 3 , on a $\operatorname{rang}\left(C_{2, L}\right)=0$, c'est à dire le nombre de classes de $L$ est impair. On a $L=\mathbb{Q}\left(\sqrt{m}, \sqrt{q^{\prime}}\right)$. Si on note par $r^{\prime}$ le nombre des premiers de $\mathbb{Q}(\sqrt{m})$ ramifiés dans $L$ et par $e^{\prime}$ l'entier associé à l'extension $L / \mathbb{Q}(\sqrt{m})$, défini $\operatorname{par} 2^{e^{\prime}}=\left[E: E \cap \mathcal{N}(L)^{*}\right]$, alors on a $\operatorname{rang}\left(C_{2, L}\right)=r^{\prime}-1-e^{\prime}=1-e^{\prime}=0$, ainsi $e^{\prime}=1$. Par suite -1 ou $\varepsilon_{m}$ n'est pas une norme dans l'extension $L / \mathbb{Q}(\sqrt{m})$. On a

$$
\left(\frac{\varepsilon_{m}, q^{\prime}}{\mathscr{P}}\right)=\left(\frac{q^{\prime}, q^{\prime}}{\mathscr{P}}\right)=\left(\frac{-1, q^{\prime}}{\mathscr{P}}\right)\left(\frac{-q^{\prime}, q^{\prime}}{\mathscr{P}}\right) .
$$

$\operatorname{Or}\left(\frac{-q^{\prime}, q^{\prime}}{\mathscr{P}}\right)=1$; alors $\left(\frac{\varepsilon_{m}, q^{\prime}}{\mathscr{P}}\right)=\left(\frac{-1, q^{\prime}}{\mathscr{P}}\right)$. Par suite $\left(\frac{-1, q^{\prime}}{\mathscr{P}}\right)=-1$, et on a $\left(\frac{-1, d}{\mathscr{P}}\right)=-1$.

Supposons que $d=2 c$ avec $c$ impair. On a

$$
\left(\frac{-1, d}{\mathscr{P}}\right)=\left(\frac{-1,2}{\mathscr{P}}\right)\left(\frac{-1, c}{\mathscr{P}}\right) \text {. }
$$

On sait que $\mathcal{N}_{\mathbb{Q}(\sqrt{m}, \sqrt{2}) / \mathbb{Q}(\sqrt{m})}\left(\varepsilon_{2}\right)=-1$, donc $\left(\frac{-1,2}{\mathscr{P}}\right)=1$. Si $c \equiv 3(\bmod 4)$, alors $\left(\frac{-1, c}{\mathscr{P}}\right)=-1$. Si $c \equiv 1(\bmod 4)$, alors $\left(\frac{-1, c}{\mathscr{P}}\right)=1$. On tire que -1 est norme dans l'extension $K / k$ si et seulement si pour tout premier $q_{1}$ de $S$ on a $\left(\frac{-1}{q_{1}}\right)=1$ et $\left[d \equiv 1(\bmod 4)\right.$ ou $d=2 c$ tel que $\left.\left(\frac{-1}{c}\right)=1\right]$.

Calculons $\left(\frac{\varepsilon_{m}, d}{\mathscr{P}}\right)$. Si $\mathscr{P}$ est au dessus d'un premier $q_{1}$ tel que $\left(\frac{m}{q_{1}}\right)=-1$, alors $\left(\frac{\varepsilon_{m}, d}{\mathscr{P}}\right)=1$. Si $\mathscr{P}$ est au dessus d'un premier $q_{1}$ tel que $q_{1} \in S$, on a $\left(\frac{\varepsilon_{m}, d}{\mathscr{P}}\right)=\left(\frac{q^{\prime}, d}{\mathscr{P}}\right)=$ $\left(\frac{a_{m}}{q_{1}}\right)$. Si $\mathscr{P}$ est au dessus de 2 , on raisonne comme suit.

On suppose que $d \equiv 3(\bmod 4)$; alors on a $\left(\frac{\varepsilon_{m}, d}{\mathscr{P}}\right)=\left(\frac{q^{\prime}, d}{\mathscr{P}}\right)=\left(\frac{q^{\prime},-d}{\mathscr{P}}\right)\left(\frac{q^{\prime},-1}{\mathscr{P}}\right)$. Or $\left(\frac{q^{\prime},-1}{\mathscr{P}}\right)=-1$ (voir le cas précédent) et $\left(\frac{q^{\prime},-d}{\mathscr{P}}\right)=1($ car $-d \equiv 1(\bmod 4))$, donc $\left(\frac{\varepsilon_{m}, d}{\mathscr{\rho}}\right)=-1$. On suppose que $d=2 c$ où $c$ est impair; alors $\left(\frac{\varepsilon_{m}, d}{\mathscr{\rho}}\right)=\left(\frac{\varepsilon_{m}, 2}{\mathscr{P}}\right)\left(\frac{\varepsilon_{m}, c}{\mathscr{\rho}}\right)$. 
Si $c \equiv 3(\bmod 4)$, alors $\left(\frac{\varepsilon_{m}, c}{\mathscr{P}}\right)=-1$. Si $c \equiv 1(\bmod 4)$, alors $\left(\frac{\varepsilon_{m}, c}{\mathscr{P}}\right)=\left(\frac{c}{\mathscr{P}}\right)^{v_{\mathscr{P}}\left(\varepsilon_{m}\right)}=1$. On a $\left(\frac{\varepsilon_{m}, 2}{\mathscr{P}}\right)=\left(\frac{q^{\prime}, 2}{\mathscr{P}}\right)=\left(\frac{2}{q^{\prime}}\right)$, d'où $\left(\frac{\varepsilon_{m}, d}{\mathscr{P}}\right)=\left(\frac{-1}{c}\right)\left(\frac{2}{q^{\prime}}\right)$. Ainsi $\varepsilon_{m}$ est norme dans l'extension $K / \mathbb{Q}(\sqrt{m})$ si et seulement si pour tout premier $q_{1} \in S$, on a $\left(\frac{a_{m}}{q_{1}}\right)=1$ et $\left[d \equiv 1(\bmod 4)\right.$ ou $d=2 c$ tel que $\left.\left(\frac{-1}{c}\right)=\left(\frac{2}{q^{\prime}}\right)\right]$. Par conséquent, on retrouve le théorème.

\section{Capitulation des 2-classes d'idéaux de $K$ où $K / \mathbb{Q}\left(\sqrt{d_{1} d_{2}}\right)$ est non ramifiée}

Soient $d_{1}$ et $d_{2}$ deux entiers naturels premiers entre eux et $K=\mathbb{Q}\left(\sqrt{d_{1}}, \sqrt{d_{2}}\right)$. On suppose que l'extension $K / \mathbb{Q}\left(\sqrt{d_{1} d_{2}}\right)$ est non ramifiée, que $\left[K^{(*)}: K\right]=2$ et que $\operatorname{Gal}\left(K_{2}^{(1)} / K\right) \simeq \mathbb{Z} / 2 \mathbb{Z} \times \mathbb{Z} / 2 \mathbb{Z}$. Alors, on donne une méthode générale qui permet de déterminer les conditions nécessaires et suffisantes sur $d_{1}$ et $d_{2}$ pour que $K_{2}^{(1)} \neq K_{2}^{(2)}$ et on donne un moyen qui permet de chercher la structure du groupe $G$. On termine par donner une application aux résultats trouvés.

Étude des conditions pour lesquels $K_{2}^{(1)} \neq K_{2}^{(2)}$. On note par $C_{\mathbb{Q}\left(\sqrt{d_{1} d_{2}}\right)}$ le 2groupe de classes du corps $\mathbb{Q}\left(\sqrt{d_{1} d_{2}}\right)$.

Théorème 5. On garde les notations précédentes et on suppose que l'extension $K / \mathbb{Q}\left(\sqrt{d_{1} d_{2}}\right)$ est non ramifiée, $\left[K^{(*)}: K\right]=2$ et $\mathrm{Gal}\left(K_{2}^{(1)} / K\right) \simeq \mathbb{Z} / 2 \mathbb{Z} \times \mathbb{Z} / 2 \mathbb{Z}$. Alors $C_{\mathbb{Q}\left(\sqrt{d_{1} d_{2}}\right)} \simeq \mathbb{Z} / 2^{2} \mathbb{Z} \times \mathbb{Z} / 2 \mathbb{Z}$ ou $C_{\mathbb{Q}\left(\sqrt{d_{1} d_{2}}\right)} \simeq \mathbb{Z} / 2 \mathbb{Z} \times \mathbb{Z} / 2 \mathbb{Z}$.

Si $C_{\mathbb{Q}\left(\sqrt{d_{1} d_{2}}\right)} \simeq \mathbb{Z} / 2^{2} \mathbb{Z} \times \mathbb{Z} / 2 \mathbb{Z}$, alors $K_{2}^{(1)}=K_{2}^{(2)}$.

Si $C_{\mathbb{Q}\left(\sqrt{d_{1} d_{2}}\right)} \simeq \mathbb{Z} / 2 \mathbb{Z} \times \mathbb{Z} / 2 \mathbb{Z}$, alors il existe un corps biquadratique L dont le 2-groupe de classes est cyclique tel que $K_{2}^{(1)} \neq K_{2}^{(2)}$ si et seulement si $8 \mid h(L)$.

Preuve. D'après [Azizi et Mouhib $\geq 2008$ ], le groupe $C_{\mathbb{Q}\left(\sqrt{d_{1} d_{2}}\right)}$ est de type $\left(2,2^{2}\right)$ ou bien de type $(2,2)$.

Si $C_{\mathbb{Q}\left(\sqrt{d_{1} d_{2}}\right)}$ est de type $\left(2,2^{2}\right)$, alors $K_{2}^{(1)}$ est le 2-corps de classes de Hilbert de $\mathbb{Q}\left(\sqrt{d_{1} d_{2}}\right)$. Comme $K$ est une extension quadratique non ramifiée de $\mathbb{Q}\left(\sqrt{d_{1} d_{2}}\right)$ dont la 2-partie du nombre de classes est égale à 4, alors d'après [Benjamin et al. 1998], la suite des 2-corps de classes de Hilbert de $\mathbb{Q}\left(\sqrt{d_{1} d_{2}}\right)$ s'arrète en $K_{2}^{(1)}$. Par conséquent on a $K_{2}^{(1)}=K_{2}^{(2)}$.

Si $C_{\mathbb{Q}\left(\sqrt{d_{1} d_{2}}\right)}$ est de type $(2,2)$, alors $K^{(*)}$ est le 2-corps de classes de Hilbert de $\mathbb{Q}\left(\sqrt{d_{1} d_{2}}\right)$. D'après la théorie des groupes, il existe une sous-extension propre de $K^{(*)} / \mathbb{Q}\left(\sqrt{d_{1} d_{2}}\right)$ notée $L$ telle que le 2-groupe de classes de $L$ est cyclique. D'autre part et comme $K^{(*)} / L$ est une extension non ramifiée, $L$ et $K^{(*)}$ ont le même 2corps de classes de Hilbert qui est $K_{2}^{(2)}$. Par suite $h\left(K_{2}^{(1)}\right)=\frac{1}{2} h\left(K^{(*)}\right)=\frac{1}{4} h(L)$. On en déduit que $K_{2}^{(1)} \neq K_{2}^{(2)}$ si et seulement si $8 \mid h(L)$, ce qui achève la preuve.

Le théorème 1 nous permet de transformer l'étude des conditions pour lesquels $K_{2}^{(1)} \neq K_{2}^{(2)}$ à une étude de la 2-partie du nombre de classes du corps $L$. Un tel corps est bien un corps biquadratique de la forme $\mathbb{Q}\left(\sqrt{d^{\prime}}, \sqrt{d^{\prime \prime}}\right)$ où $d^{\prime}$ et $d^{\prime \prime}$ sont deux entiers premiers entre eux divisant $d_{1} d_{2}$. Or $K^{(*)} / L$ est bien une extension de degré 2 et $K^{(*)}$ est le corps de genres de $L$; par suite et à l'aide de la théorie des 
genres; l'un des corps quadratiques $\mathbb{Q}\left(\sqrt{d^{\prime}}\right)$ ou $\mathbb{Q}\left(\sqrt{d^{\prime \prime}}\right)$ est de nombre de classes impair. Donc $L$ contient un corps quadratique de nombres de classes impair et par suite $L$ peut être déterminer à partir des théorèmes 1,2, 3 et 4 . Ainsi, à l'aide des résultats connus sur la 2-partie du nombre de classes des corps quadratiques (voir par exemple [Kaplan 1976; Kučera 1995; Benjamin et Snyder 1995]) et en utilisant la formule de Wada sur le nombre de classes d'un composé de corps quadratiques (voir [Wada 1966]), on peut déterminer les conditions nécessaires et suffisantes sur $d_{1}$ et $d_{2}$ pour lesquels $8 \mid h(L)$ et par suite $K_{2}^{(1)} \neq K_{2}^{(2)}$.

Structure du groupe $\boldsymbol{G}=\mathbf{G a l}\left(\boldsymbol{K}_{\mathbf{2}}^{(2)} / \boldsymbol{K}\right)$. On sait que $C_{\mathbb{Q}\left(\sqrt{d_{1} d_{2}}\right)} \simeq \mathbb{Z} / 2^{2} \mathbb{Z} \times \mathbb{Z} / 2 \mathbb{Z}$ ou $C_{\mathbb{Q}\left(\sqrt{d_{1} d_{2}}\right)} \simeq \mathbb{Z} / 2 \mathbb{Z} \times \mathbb{Z} / 2 \mathbb{Z}$.

Si $C_{\mathbb{Q}\left(\sqrt{d_{1} d_{2}}\right)} \simeq \mathbb{Z} / 2^{2} \mathbb{Z} \times \mathbb{Z} / 2 \mathbb{Z}$, alors $K_{2}^{(1)}=K_{2}^{(2)}$. Par conséquent $G$ est abélien.

Si $C_{\mathbb{Q}\left(\sqrt{d_{1} d_{2}}\right)} \simeq \mathbb{Z} / 2 \mathbb{Z} \times \mathbb{Z} / 2 \mathbb{Z}$, alors $K^{(*)}$ est le 2-corps de classes de Hilbert de $\mathbb{Q}\left(\sqrt{d_{1} d_{2}}\right)$. De plus $K_{2}^{(2)}$ est le 2 -corps de classes de Hilbert de $K^{(*)}$. On se ramène ainsi à des questions de capitulation sur des corps quadratiques. En utilisant [Benjamin et Snyder 1995] et [Couture et Derhem 1992], on trouve la structure du groupe $\operatorname{Gal}\left(K_{2}^{(2)} / \mathbb{Q}\left(\sqrt{d_{1} d_{2}}\right)\right)$. Comme $G$ est un sous-groupe d'indice 2 dans $\operatorname{Gal}\left(K_{2}^{(2)} / \mathbb{Q}\left(\sqrt{d_{1} d_{2}}\right)\right)$, la structure du groupe $G$ est bien déterminée.

Dans la suite on va donner une application à cette étude pour illustrer la méthode qu'on vient de décrire.

Soient $p, p^{\prime}, q_{1}, q_{2}$ des premiers différents tels que $p \equiv p^{\prime} \equiv-q_{1} \equiv-q_{2} \equiv 1$ $(\bmod 4)$ et $K=\mathbb{Q}\left(\sqrt{d_{1}}, \sqrt{d_{2}}\right)$ où $d_{1}=p$ et $d_{2}=p^{\prime} q_{1} q_{2}$. Les entiers naturels $d_{1}$ et $d_{2}$ sont premiers entre eux et on a $K^{(*)}=\mathbb{Q}\left(\sqrt{p}, \sqrt{p^{\prime}}, \sqrt{q_{1} q_{2}}\right)$ et par suite $\left[K^{(*)}: K\right]=2$.

Théorème 6 [Azizi et Mouhib 2001]. Soient $p, p^{\prime}, q_{1}, q_{2}$ des premiers différents tels que $p \equiv p^{\prime} \equiv-q_{1} \equiv-q_{2} \equiv 1(\bmod 4)$ et $K=\mathbb{Q}\left(\sqrt{p}, \sqrt{p^{\prime} q_{1} q_{2}}\right)$. Alors le 2-groupe de classes de $K$ est de type $(2,2)$ si et seulement si l'un des cas suivants est vérifié:

1) $\left(\frac{p}{p^{\prime}}\right)=-\left(\frac{p}{q_{1}}\right)=-\left(\frac{p}{q_{2}}\right)=1$ et $\left(\frac{p^{\prime}}{q_{1}}\right)\left(\frac{p^{\prime}}{q_{2}}\right)=-1$.

2) $-\left(\frac{p}{p^{\prime}}\right)=\left(\frac{p}{q_{1}}\right)=\left(\frac{p}{q_{2}}\right)=1$ et $\left(\frac{p^{\prime}}{q_{1}}\right)\left(\frac{p^{\prime}}{q_{2}}\right)=-1$.

3) $-\left(\frac{p}{p^{\prime}}\right)=\left(\frac{p}{q_{1}}\right)=\left(\frac{p}{q_{2}}\right)=1$ et $\left(\frac{p^{\prime}}{q_{1}}\right)=\left(\frac{p^{\prime}}{q_{2}}\right)=-1$.

4) $\left(\frac{p}{p^{\prime}}\right)=1,\left(\frac{p}{q_{1}}\right)\left(\frac{p}{q_{2}}\right)=-1,\left(\frac{p^{\prime}}{q_{1}}\right)\left(\frac{p^{\prime}}{q_{2}}\right)=-1$ et $\left(\frac{p}{q_{1}}\right)=\left(\frac{p^{\prime}}{q_{1}}\right)$.

5) $\left(\frac{p}{p^{\prime}}\right)=1,\left(\frac{p}{q_{1}}\right)\left(\frac{p}{q_{2}}\right)=-1,\left(\frac{p}{q_{1}}\right) \neq\left(\frac{p^{\prime}}{q_{1}}\right)$ et $\left[\left(\frac{p^{\prime}}{q_{1}}\right)=-1\right.$ ou $\left.\left(\frac{p^{\prime}}{q_{2}}\right)=-1\right]$.

Dans ce qui suit, on va étudier le problème de capitulation des 2-classes d'idéaux de $K$, en distinguant les cinq cas du théorème 6 . 
Étude des conditions pour lesquels $K_{2}^{(1)} \neq K_{2}^{(2)}$. Nous aurons besoin de quelques résultats sur les unités qu'on va démontrer dans ce qui suit:

Soient $L=\mathbb{Q}(\sqrt{A}, \sqrt{B})$ un corps biquadratique réel et $Q_{L}$ l'indice du groupe des unités de $L$ modulo son sous-groupe engendré par les unités des trois souscorps quadratiques de $L$. On suppose que $\operatorname{Gal}(L / \mathbb{Q})$ est engendré par $\sigma$ et $\tau$ et que $\mathbb{Q}(\sqrt{A})=\operatorname{inv}(\sigma), \mathbb{Q}(\sqrt{B})=\operatorname{inv}(\tau)$ et $\mathbb{Q}(\sqrt{A B})=\operatorname{inv}(\sigma \tau)$. D'après une idée qui revient à Hergoltz, on sait que le carré d'une unité $\varepsilon$ de $L$ vérifie

$$
\varepsilon^{2}=\left(\varepsilon \varepsilon^{\sigma}\right)\left(\varepsilon \varepsilon^{\tau}\right)\left(\varepsilon^{\sigma} \varepsilon^{\tau}\right)^{-1},
$$

c'est-à-dire il est un produit d'unités dans $\mathbb{Q}(\sqrt{A})$, dans $\mathbb{Q}(\sqrt{B})$ et dans $\mathbb{Q}(\sqrt{A B})$ (voire $\varepsilon \varepsilon^{\sigma}, \varepsilon \varepsilon^{\tau}, \varepsilon^{\sigma} \varepsilon^{\tau}$ respectivement). Ainsi, l'indice $Q_{L}$ est égal à 1, 2, 4 ou 8.

T. Kubota [1956] a démontré que $Q_{L} \in\{1,2,4\}$. S'il existe exactement $r$ relations quadratiques et indépendantes entre les unités $\varepsilon_{1}$, $\varepsilon_{2}$ et $\varepsilon_{3}$ des trois sous-corps quadratiques de $L$, alors $Q_{L}=2^{r}$.

Pour $m$ entier naturel, on désigne dans tout ce quit suit par $e(m)$ la norme par rapport à l'extension $\mathbb{Q}(\sqrt{m}) / \mathbb{Q}$ de l'unité fondamentale de $\mathbb{Q}(\sqrt{m})$.

Lemme 1. Soient $q_{1}, q_{2}, p, p^{\prime}$ des premiers différents tels que $p \equiv p^{\prime} \equiv-q_{1} \equiv$ $-q_{2} \equiv 1(\bmod 4)$ et $L=\mathbb{Q}\left(\sqrt{q_{1} q_{2}}, \sqrt{p p^{\prime}}\right)$. On suppose que e $\left(p p^{\prime}\right)=1$; alors $Q_{L}=2$.

Preuve. Soit $\varepsilon_{1}$ l'unité fondamentale de $\mathbb{Q}\left(\sqrt{q_{1} q_{2}}\right), \varepsilon_{2}$ l'unité fondamentale de $\mathbb{Q}\left(\sqrt{p p^{\prime}}\right)$ et $\varepsilon_{3}$ l'unité fondamentale de $\mathbb{Q}\left(\sqrt{p p^{\prime} q_{1} q_{2}}\right)$. Comme $e\left(q_{1} q_{2}\right)=e\left(p p^{\prime}\right)=$ $e\left(p p^{\prime} q_{1} q_{2}\right)=1$, on a, d'après [Cohn 1978, théorème 11.23, p. 106], les trois relations suivantes:

$$
\begin{aligned}
& \sqrt{\varepsilon_{1}}=y_{1} \sqrt{q_{1}}+y_{2} \sqrt{q_{2}}, \quad \text { avec } y_{1}, y_{2} \in \mathbb{Q} ; \\
& \sqrt{\varepsilon_{2}}=t_{1} \sqrt{p}+t_{2} \sqrt{p^{\prime}}, \quad \text { avec } t_{1}, t_{2} \in \mathbb{Q} \text {; } \\
& \sqrt{\varepsilon_{3}}=v_{1} \sqrt{m}+v_{2} \sqrt{n}, \quad \text { avec } v_{1}, v_{2} \in \mathbb{Q}, m, n \in \mathbb{Z}, m n=p p^{\prime} q_{1} q_{2} .
\end{aligned}
$$

Donc, il existe trois entiers naturels uniques $i, j, k$ tels que $\{i, j, k\} \subset\{0,1\}$, $i+j+k \neq 0$ et $\sqrt{\varepsilon_{1}^{i} \varepsilon_{2}^{j} \varepsilon_{3}^{k}} \in K$. Par conséquent $Q_{L}=2$.

Lemme 2. Soient $p, p^{\prime}, q_{1}, q_{2}$ des premiers différents tels que $p \equiv p^{\prime} \equiv-q_{1} \equiv$ $-q_{2} \equiv 1(\bmod 4)$ et $L=\mathbb{Q}\left(\sqrt{q_{1} q_{2}}, \sqrt{p p^{\prime}}\right)$. On suppose que $e\left(p p^{\prime}\right)=-1$,

$$
\left(\frac{p^{\prime}}{q_{1}}\right)\left(\frac{p^{\prime}}{q_{2}}\right)=-1 \quad \text { et } \quad\left(\frac{p}{p^{\prime}}\right)=-\left(\frac{p}{q_{1}}\right)=-\left(\frac{p}{q_{2}}\right)=1 \text {. }
$$

Alors $Q_{L}=1$.

Preuve. Comme $e\left(p p^{\prime}\right)=-1$, l'unité fondamentale de $\mathbb{Q}\left(\sqrt{p p^{\prime}}\right)$ n'est pas un carré dans $L$. Soient $\varepsilon_{1}$ et $\varepsilon_{2}$ les unités fondamentales respectives des corps $\mathbb{Q}\left(\sqrt{q_{1} q_{2}}\right)$ et $\mathbb{Q}\left(\sqrt{p p^{\prime} q_{1} q_{2}}\right)$. On sait d'après la preuve du lemme 1 qu'il existe deux nombres rationnels $y_{1}$ et $y_{2}$ tels que

$$
\sqrt{\varepsilon_{1}}=y_{1} \sqrt{q_{1}}+y_{2} \sqrt{q_{2}} .
$$


Soit $w$ un entier naturel sans facteurs carrés tel que $\mathcal{N}_{\mathbb{Q}\left(\sqrt{p p^{\prime} q_{1} q_{2}}\right) / \mathbb{Q}}\left(1+\varepsilon_{2}\right)=w x^{2}$ où $x$ est un entier naturel. D'après [Benjamin et Snyder 1995], on a $w=p^{\prime} q_{1} q_{2}$; par suite il existe deux entiers naturels $b$ et $c$ tels que

$$
\sqrt{\varepsilon_{2}}=\frac{c}{2} \sqrt{p^{\prime} q_{1} q_{2}}+\frac{b}{2} \sqrt{p}
$$

De cela et de (3-1) on tire que les unités $\varepsilon_{1}$, $\varepsilon_{2}$ et $\varepsilon_{1} \varepsilon_{2}$ ne sont pas des carrés dans $L$. Par conséquent $Q_{L}=1$.

Lemme 3. Soient $p, p^{\prime}, q_{1}, q_{2}$ des premiers différents tels que $p \equiv p^{\prime} \equiv-q_{1} \equiv$ $-q_{2} \equiv 1(\bmod 4)$ et $L=\mathbb{Q}\left(\sqrt{q_{1} q_{2}}, \sqrt{p p^{\prime}}\right)$. On suppose que e $\left(p p^{\prime}\right)=-1,\left(\frac{p}{p^{\prime}}\right)=1$, $\left(\frac{p}{q_{1}}\right)\left(\frac{p}{q_{2}}\right)=-1,\left(\frac{p^{\prime}}{q_{1}}\right)\left(\frac{p^{\prime}}{q_{2}}\right)=-1$, et $\left(\frac{p}{q_{1}}\right)=\left(\frac{p^{\prime}}{q_{1}}\right) ;$ alors $Q_{L}=2$.

Preuve. Soient $\varepsilon_{1}$ et $\varepsilon_{2}$ les unités fondamentales respectives des corps $\mathbb{Q}\left(\sqrt{q_{1} q_{2}}\right)$ et $\mathbb{Q}\left(\sqrt{p p^{\prime} q_{1} q_{2}}\right)$. D'après la preuve du lemme 1 , il existe deux nombres rationnels $y_{1}$ et $y_{2}$ tels que

$$
\sqrt{\varepsilon_{1}}=y_{1} \sqrt{q_{1}}+y_{2} \sqrt{q_{2}} .
$$

Soit $w$ l'entier naturel défini dans la preuve du lemme 2. D'après [Benjamin et Snyder 1995], on a $w=q_{1}$ ou $w=q_{2}$; par suite il existe deux entiers naturels $c$ et $d$ tels que

$$
\sqrt{\varepsilon_{2}}=\frac{c}{2} \sqrt{w}+\frac{d}{2} \sqrt{\frac{p p^{\prime} q_{1} q_{2}}{w}}
$$

Par suite, on a $\sqrt{\varepsilon_{1} \varepsilon_{2}} \in L$, d'où $Q_{L}=2$.

Lemme 4. Soient $q$, $p$ deux premiers différents tels que $p \equiv-q \equiv 1(\bmod 4)$ et $L=\mathbb{Q}(\sqrt{q}, \sqrt{2 p})$. Alors :

1) $e(2 p)=1 \Longrightarrow Q_{L}=4$.

2) $e(2 p)=-1 \Longrightarrow Q_{L}=2$.

Preuve. 1) Soient $\varepsilon_{1}, \varepsilon_{2}$ et $\varepsilon_{3}$ les unités fondamentales de $\mathbb{Q}(\sqrt{q}), \mathbb{Q}(\sqrt{2 p})$ et $\mathbb{Q}(\sqrt{2 p q})$ respectivement. On vérifie facilement qu'il existe deux nombres rationnels $a_{1}$ et $a_{2}$ tels que

$$
\sqrt{\varepsilon_{1}}=a_{1} \sqrt{2}+a_{2} \sqrt{2 q} .
$$

Comme $e(2 p)=1$, il existe deux nombres rationnels $b_{1}$ et $b_{2}$ tels que

$$
\sqrt{\varepsilon_{2}}=b_{1} \sqrt{2}+b_{2} \sqrt{p} \text {. }
$$

D'autre part il existe deux nombres rationnels $c_{1}$ et $c_{2}$ tels que

$$
\sqrt{\varepsilon_{3}}=c_{1} \sqrt{m}+c_{2} \sqrt{n}
$$

où $m$ et $n$ sont deux entiers naturels premiers entre eux tels que $m n=2 p q$.

De (3-2) et (3-3), on déduit que $\sqrt{\varepsilon_{1} \varepsilon_{2}} \in L$ et de (3-2) et (3-4), on déduit que $\sqrt{\varepsilon_{3}} \in L$ ou bien $\sqrt{\varepsilon_{1} \varepsilon_{3}} \in L$. Par conséquent, on a $Q_{L}=4$.

2) On suppose que $e(2 p)=-1$. D'après 1$)$, on a $\sqrt{\varepsilon_{1}} \notin L$ et $\sqrt{\varepsilon_{3}} \in L$ ou bien $\sqrt{\varepsilon_{1} \varepsilon_{3}} \in L$. Par suite on a $Q_{L}=2$. 
Lemme 5. Soient $p, q, q^{\prime}$ des premiers différents tels que $p \equiv-q \equiv-q^{\prime} \equiv 1$ $(\bmod 4)$ et $K=\mathbb{Q}\left(\sqrt{q}, \sqrt{p q^{\prime}}\right)$. On suppose que $\left(\frac{q^{\prime}}{p}\right)=-\left(\frac{q}{p}\right)=1 ;$ alors $Q_{K}=2$. Preuve. Soit $\varepsilon_{1}$ l'unité fondamentale de $\mathbb{Q}(\sqrt{q})$. Comme $e(q)=1$, il existe deux nombres rationnels $t_{1}$ et $t_{2}$ tels que

$$
\sqrt{\varepsilon_{1}}=t_{1} \sqrt{2}+t_{2} \sqrt{2 q} \text {. }
$$

Soit $\varepsilon_{2}$ l'unité fondamentale de $\mathbb{Q}\left(\sqrt{p q^{\prime}}\right)$. Comme $e\left(p q^{\prime}\right)=1$, il existe deux nombres rationnels $v_{1}$ et $v_{2}$ tels que

$$
\sqrt{\varepsilon_{2}}=v_{1} \sqrt{m}+v_{2} \sqrt{n},
$$

et $m, n$ deux entiers naturels tels que $m n \in\left\{p q^{\prime}, 4 p q^{\prime}\right\}$.

Soit $\varepsilon_{3}=\frac{x}{2}+\frac{y}{2} \sqrt{p q q^{\prime}}$ où $x$ et $y$ sont des entiers, l'unité fondamentale de $\mathbb{Q}\left(\sqrt{p q q^{\prime}}\right)$. On a $e\left(p q q^{\prime}\right)=1$; alors $(x-2)(x+2)=p q q^{\prime} y^{2}$. Comme $\left(\frac{q^{\prime}}{p}\right)=$ $-\left(\frac{q}{p}\right)=1$, il existe deux nombres rationnels $y_{1}$ et $y_{2}$ tels que

$$
\left\{\begin{array}{l}
x \pm 2=q^{\prime} y_{1}^{2} \\
x \mp 2=p q y_{2}^{2} .
\end{array} \text { où } 2 y_{1} y_{2}=y\right.
$$

En posant $W=\sqrt{q^{\prime}} y_{1}+\sqrt{p q} y_{2}$, on trouve que $W^{2}=4 \varepsilon_{3}$. Ainsi

$$
\sqrt{\varepsilon_{3}}=\frac{y_{1}}{2} \sqrt{q^{\prime}}+\frac{y_{2}}{2} \sqrt{p q} .
$$

De (3-5), (3-6) et (3-7), on a $\sqrt{\varepsilon_{1}} \notin K, \sqrt{\varepsilon_{3}} \notin K$. De plus, il existe trois entiers naturels $i, j, k$ uniques tels que $\{i, j, k\} \subset\{0,1\}, i+j+k \neq 0$ et $\sqrt{\varepsilon_{1}^{i} \varepsilon_{2}^{j} \varepsilon_{3}^{k}} \in K$. Ainsi, on a $Q_{K}=2$.

Théorème 7. Soient $p, p^{\prime}, q_{1}, q_{2}$ des premiers différents tels que $p \equiv p^{\prime} \equiv-q_{1} \equiv$ $-q_{2} \equiv 1(\bmod 4)$ et $K=\mathbb{Q}\left(\sqrt{p}, \sqrt{p^{\prime} q_{1} q_{2}}\right)$.

- On suppose que $K$ vérifie les conditions des cas 1 ou 5 du théorème 6; alors $L=\mathbb{Q}\left(\sqrt{q_{1} q_{2}}, \sqrt{p p^{\prime}}\right)$ et $K_{2}^{(1)} \neq K_{2}^{(2)}$ si et seulement si $\left(\frac{p}{p^{\prime}}\right)_{4}=\left(\frac{p^{\prime}}{p}\right)_{4}=1$.

- On suppose que $K$ vérifie les conditions du cas $2 d u$ théorème 6; alors $L=$ $\mathbb{Q}\left(\sqrt{p^{\prime}}, \sqrt{p q_{1} q_{2}}\right)$ et $K_{2}^{(1)} \neq K_{2}^{(2)}$ si et seulement si $8 \mid h\left(p q_{1} q_{2}\right)$ ou $\left[Q_{L}=2\right.$ et $\left.h\left(p q_{1} q_{2}\right) \equiv 4(\bmod 8)\right]$.

- On suppose que $K$ vérifie les conditions du cas 3 du théorème 6; alors $L=$ $\mathbb{Q}\left(\sqrt{p^{\prime}}, \sqrt{p q_{1} q_{2}}\right)$ et $K_{2}^{(1)} \neq K_{2}^{(2)}$ si et seulement si $8 \mid h\left(p q_{1} q_{2}\right)$ ou $\left[Q_{L}=2\right.$ et $\left.h\left(p q_{1} q_{2}\right) \equiv 4(\bmod 8)\right]$.

- On suppose que $K$ vérifie les conditions du cas 4 du théorème 6; alors $L=$ $\mathbb{Q}\left(\sqrt{q_{1} q_{2}}, \sqrt{p p^{\prime}}\right)$ et $K_{2}^{(1)} \neq K_{2}^{(2)}$ si et seulement si $\left(\frac{2}{p}\right)_{4}=(-1)^{(p-1) / 8}$.

Preuve. On note par $\varepsilon_{1}$ l'unité fondamentale de $\mathbb{Q}\left(\sqrt{q_{1} q_{2}}\right)$ et par $\varepsilon_{2}$ l'unité fondamentale de $\mathbb{Q}\left(\sqrt{p p^{\prime} q_{1} q_{2}}\right)$. Soit $w$ un entier naturel sans facteurs carrés tel que $\mathcal{N}_{\mathbb{Q}\left(\sqrt{p p^{\prime} q_{1} q_{2}}\right) / \mathbb{Q}}\left(1+\varepsilon_{2}\right)=w x^{2}$ où $x$ est un entier naturel. 
- On suppose que $K$ vérifie les conditions du cas 1 du théorème 6. Donc

$$
\left(\frac{p}{p^{\prime}}\right)=-\left(\frac{p}{q_{1}}\right)=-\left(\frac{p}{q_{2}}\right)=1 \quad \text { et } \quad\left(\frac{p^{\prime}}{q_{1}}\right)\left(\frac{p^{\prime}}{q_{2}}\right)=-1 .
$$

D'après le théorème 4 , le 2 -groupe de classes de $L=\mathbb{Q}\left(\sqrt{q_{1} q_{2}}, \sqrt{p p^{\prime}}\right)$ est cyclique. On sait d'après le théorème 5 que $K_{2}^{(1)} \neq K_{2}^{(2)}$ si et seulement si $8 \mid h(L)$. D'après [Kaplan 1976], $h\left(p p^{\prime} q_{1} q_{2}\right) \equiv 4(\bmod 8)$ et $e\left(p p^{\prime} q_{1} q_{2}\right)=1$. Si $\left(\frac{p}{p^{\prime}}\right)_{4} \neq\left(\frac{p^{\prime}}{p}\right)_{4}$, alors, d'après [Kučera 1995], on a $h\left(p p^{\prime}\right) \equiv 2(\bmod 4)$ et $e\left(p p^{\prime}\right)=1$. D'autre part, d'après le lemme 1 , on a $Q_{L}=2$, par suite, d'après [Wada 1966], on a

$$
h(L)=\frac{Q_{L} h\left(q_{1} q_{2}\right) h\left(p p^{\prime}\right) h\left(p p^{\prime} q_{1} q_{2}\right)}{4} \equiv 4(\bmod 8) .
$$

Ainsi on a $K_{2}^{(1)}=K_{2}^{(2)}$. Si $\left(\frac{p}{p^{\prime}}\right)_{4}=\left(\frac{p^{\prime}}{p}\right)_{4}=-1$, alors d'après [Kučera 1995], on a $h\left(p p^{\prime}\right) \equiv 4(\bmod 8)$ et $e\left(p p^{\prime}\right)=-1$. D'autre part, d'après le lemme 2 , on a $Q_{L}=1$ et donc $h(L) \equiv 4(\bmod 8)$. $S i\left(\frac{p}{p^{\prime}}\right)_{4}=\left(\frac{p^{\prime}}{p}\right)_{4}=1$, alors si $e\left(p p^{\prime}\right)=-1$, on a $8 \mid h\left(p p^{\prime}\right)$, d'après [Kučera 1995]; par suite $8 \mid h(L)$. Ainsi, on a $K_{2}^{(1)} \neq K_{2}^{(2)}$. Si $e\left(p p^{\prime}\right)=1$, alors, d'après le lemme 1 , on a $Q_{L}=2$; par suite $8 \mid h(L)$. Donc

$$
K_{2}^{(1)} \neq K_{2}^{(2)} \Longleftrightarrow\left(\frac{p}{p^{\prime}}\right)_{4}=\left(\frac{p^{\prime}}{p}\right)_{4}=1 \text {. }
$$

- On suppose que $K$ vérifie les conditions du cas 2 du théorème 6. Donc

$$
-\left(\frac{p}{p^{\prime}}\right)=\left(\frac{p}{q_{1}}\right)=\left(\frac{p}{q_{2}}\right)=1 \quad \text { et } \quad\left(\frac{p^{\prime}}{q_{1}}\right)\left(\frac{p^{\prime}}{q_{2}}\right)=-1 .
$$

D'après le théorème 1 , le 2-groupe de classes de $L=\mathbb{Q}\left(\sqrt{p^{\prime}}, \sqrt{p q_{1} q_{2}}\right)$ est cyclique. Or, d'après [Kaplan 1976], on a $h\left(p p^{\prime} q_{1} q_{2}\right) \equiv 4(\bmod 8)$ et $4 \mid h\left(p q_{1} q_{2}\right)$, donc on a

$$
K_{2}^{(1)} \neq K_{2}^{(2)} \Longleftrightarrow 8 \mid h\left(p q_{1} q_{2}\right) \text { ou bien }\left[h\left(p q_{1} q_{2}\right) \equiv 4(\bmod 8) \text { et } Q_{L}=2\right] \text {. }
$$

- On suppose que $K$ vérifie les conditions du cas 3 du théorème 6. Donc

$$
-\left(\frac{p}{p^{\prime}}\right)=\left(\frac{p}{q_{1}}\right)=\left(\frac{p}{q_{1}}\right)=1 \quad \text { et } \quad\left(\frac{p^{\prime}}{q_{1}}\right)=\left(\frac{p^{\prime}}{q_{2}}\right)=-1 .
$$

D'après le théorème 1 , le 2-groupe de classes de $L=\mathbb{Q}\left(\sqrt{p^{\prime}}, \sqrt{p q_{1} q_{2}}\right)$ est cyclique. Ce cas est similaire au cas 2; par suite, on a

$$
K_{2}^{(1)} \neq K_{2}^{(2)} \Longleftrightarrow 8 \mid h\left(p q_{1} q_{2}\right) \text { ou bien }\left[h\left(p q_{1} q_{2}\right) \equiv 4(\bmod 8) \text { et } Q_{L}=2\right] .
$$

- On suppose que $K$ vérifie les conditions du cas 4 du théorème 6. Donc

$$
\left(\frac{p}{p^{\prime}}\right)=1, \quad\left(\frac{p}{q_{1}}\right)\left(\frac{p}{q_{2}}\right)=-1, \quad\left(\frac{p^{\prime}}{q_{1}}\right)\left(\frac{p^{\prime}}{q_{2}}\right)=-1, \quad\left(\frac{p}{q_{1}}\right)=\left(\frac{p^{\prime}}{q_{1}}\right) .
$$

D'après le théorème 4 , le 2-groupe de classes de $L=\mathbb{Q}\left(\sqrt{q_{1} q_{2}}, \sqrt{p p^{\prime}}\right)$ est cyclique. D'après [Kaplan 1976], on a $h\left(p p^{\prime} q_{1} q_{2}\right) \equiv 4(\bmod 8)$. Si $\left(\frac{p}{p^{\prime}}\right)_{4} \neq\left(\frac{p^{\prime}}{p}\right)_{4}$, on trouve que $K_{2}^{(1)}=K_{2}^{(2)}$, comme dans le cas 1 du théorème 6 . Dans le cas contraire, d'après [Kučera 1995], on a $4 \mid h\left(p p^{\prime}\right)$ et on distingue les cas suivants:

Si $e\left(p p^{\prime}\right)=-1$, d'après le lemme 3 , on a $Q_{L}=2$; par suite $K_{2}^{(1)} \neq K_{2}^{(2)}$.

Si $e\left(p p^{\prime}\right)=1$, d'après le lemme 1 , on a $Q_{L}=2$; par suite $8 \mid h(L)$. Par conséquent

$$
K_{2}^{(1)} \neq K_{2}^{(2)} \Longleftrightarrow\left(\frac{p}{p^{\prime}}\right)_{4}=\left(\frac{p^{\prime}}{p}\right)_{4} .
$$


- On suppose que $K$ vérifie les conditions du cas 5 du théorème 6. Donc

$$
\left(\frac{p}{p^{\prime}}\right)=1, \quad\left(\frac{p}{q_{1}}\right)\left(\frac{p}{q_{2}}\right)=-1, \quad\left(\frac{p}{q_{1}}\right) \neq\left(\frac{p^{\prime}}{q_{1}}\right), \quad\left[\left(\frac{p^{\prime}}{q_{1}}\right)=-1 \text { ou }\left(\frac{p^{\prime}}{q_{2}}\right)=-1\right] .
$$

D'après le théorème 4 , le 2-groupe de classes de $L=\mathbb{Q}\left(\sqrt{q_{1} q_{2}}, \sqrt{p p^{\prime}}\right)$ est cyclique. Ce cas est similaire au cas 1 , donc on a

$$
K_{2}^{(1)} \neq K_{2}^{(2)} \Longleftrightarrow\left(\frac{p}{p^{\prime}}\right)_{4}=\left(\frac{p^{\prime}}{p}\right)_{4}=1 .
$$

Structure du groupe $\boldsymbol{G}=\boldsymbol{G a l}\left(\boldsymbol{K}_{\mathbf{2}}^{(2)} / \boldsymbol{K}\right)$. On a prouvé précédemment (page 23) que la détermination de la structure du groupe $G$ revient à chercher la structure du groupe $\operatorname{Gal}\left(K_{2}^{(2)} / \mathbb{Q}(\sqrt{2 d})\right)$. Alors en utilisant [Couture et Derhem 1992] et [Benjamin et Snyder 1995], on retrouve le théorème suivant: On désigne par $w$ l'entier naturel sans facteurs carrés défini par $\mathcal{N}_{\mathbb{Q}\left(\sqrt{p q_{1} q_{2}}\right) / \mathbb{Q}}\left(1+\varepsilon_{p q_{1} q_{2}}\right)=w n^{2}$ où $\varepsilon_{p q_{1} q_{2}}$ est l'unité fondamentale de $\mathbb{Q}\left(\sqrt{p q_{1} q_{2}}\right)$ et $n$ un entier naturel.

Théorème 8. Soient $p, p^{\prime}, q_{1}, q_{2}$ des premiers différents tels que $p \equiv p^{\prime} \equiv-q_{1} \equiv$ $-q_{2} \equiv 1(\bmod 4)$ et $K=\mathbb{Q}\left(\sqrt{p}, \sqrt{p^{\prime} q_{1} q_{2}}\right)$.

- On suppose que $K$ vérifie les conditions du cas 1 du théorème 6 et $K_{2}^{(1)} \neq K_{2}^{(2)}$; alors $G$ est diédral.

- On suppose que $K$ vérifie les conditions du cas 2 du théorème 6 et $K_{2}^{(1)} \neq K_{2}^{(2)}$; alors $G$ est quaternionique si $w \in\left\{p q_{1}, p q_{2}\right\}$, sinon $G$ est diédral.

- On suppose que $K$ vérifie les conditions du cas 3 du théorème 6 et $K_{2}^{(1)} \neq K_{2}^{(2)}$; alors $G$ est quaternionique si $w=p$, sinon $G$ est diédral.

- On suppose que $K$ vérifie les conditions du cas 4 du théorème 6 et $K_{2}^{(1)} \neq K_{2}^{(2)}$; alors $G$ est quaternionique si e $(2 p)=-1$, sinon $G$ est diédral.

- On suppose que $K$ vérifie les conditions du cas 5 du théorème 6 et $K_{2}^{(1)} \neq K_{2}^{(2)}$; alors $G$ est diédral.

Preuve. On utilise plusieurs fois des résultats contenus dans [Benjamin et Snyder 1995].

Dans le cas 1 du théorème 6 , on a $h\left(p p^{\prime} q_{1} q_{2}\right) \equiv 4(\bmod 8)$. Si $K_{2}^{(1)} \neq K_{2}^{(2)}$, alors $\operatorname{Gal}\left(K_{2}^{(2)} / Q\left(\sqrt{p p^{\prime} q_{1} q_{2}}\right)\right)$ est diédral et comme $G$ est est un sous-groupe d'indice 2 dans $\operatorname{Gal}\left(K_{2}^{(2)} / Q\left(\sqrt{p p^{\prime} q_{1} q_{2}}\right)\right)$, alors $G$ est diédral.

Dans le cas 2 du théorème 6 , on a $h\left(p p^{\prime} q_{1} q_{2}\right) \equiv 4(\bmod 8)$. Si $K_{2}^{(1)} \neq K_{2}^{(2)}$, alors $\operatorname{Gal}\left(K_{2}^{(2)} / Q\left(\sqrt{p p^{\prime} q_{1} q_{2}}\right)\right)$ est quaternionique si $w \in\left\{p q_{1}, p q_{2}\right\}$, et diédral dans le cas contraire. $G$ est d'indice 2 dans $\operatorname{Gal}\left(K_{2}^{(2)} / Q\left(\sqrt{p p^{\prime} q_{1} q_{2}}\right)\right.$ ), ce qui donne le résultat.

Pour les autres cas du théorème 6 , on a toujours $h\left(2 p q_{1} q_{2}\right) \equiv 4(\bmod 8)$, et on trouve le résultat toujours en utilisant [Benjamin et Snyder 1995].

\section{Capitulation des 2-classes d'idéaux de $K$ où $K / \mathbb{Q}\left(\sqrt{d_{1} d_{2}}\right)$ est ramifiée}

Dans cette section, on suppose que $\operatorname{Gal}\left(K_{2}^{(1)} / K\right) \simeq \mathbb{Z} / 2 \mathbb{Z} \times \mathbb{Z} / 2 \mathbb{Z},\left[K^{(*)}: K\right]=2$ et l'extension $K / \mathbb{Q}\left(\sqrt{d_{1} d_{2}}\right)$ est ramifiée. D'après [Azizi et Mouhib $\geq 2008$ ], la 2partie du nombre de classes de $\mathbb{Q}\left(\sqrt{d_{1} d_{2}}\right)$ est égale à 1 ou à 2 ; par suite la 2-partie 
du nombre de classes de $\mathbb{Q}\left(\sqrt{d_{1} d_{2}}\right)$ est égale à 2 (car sinon le nombre de classes de $K$ serait impair). D'après [Kaplan 1976], les cas tels que $h\left(d_{1} d_{2}\right) \equiv 2(\bmod 4)$ sont:

1) $d_{1} d_{2}=2 p q$ où $p \equiv-q \equiv 1(\bmod 4)$ et $\left(\frac{2}{p}\right)=-1$ ou $\left(\frac{q}{p}\right)=-1$.

2) $d_{1} d_{2}=p q q^{\prime}$ où $p \equiv-q \equiv-q^{\prime} \equiv 1(\bmod 4)$ et $\left(\frac{q}{p}\right)=-1$ ou $\left(\frac{q^{\prime}}{p}\right)=-1$.

3) $d_{1} d_{2}=p p^{\prime}$ où $p \equiv p^{\prime} \equiv 1(\bmod 4)$ et $h\left(p p^{\prime}\right) \equiv 2(\bmod 4)$.

4) $d_{1} d_{2}=2 p$ où $p \equiv 1(\bmod 4)$ et $h(2 p) \equiv 2(\bmod 4)$.

5) $d_{1} d_{2}=2 q q^{\prime}$ où $q \equiv q^{\prime} \equiv-1(\bmod 4)$ et $h\left(2 q q^{\prime}\right) \equiv 2(\bmod 4)$.

6) $d_{1} d_{2}=p q$ où $p \equiv-q \equiv 1(\bmod 4)$ et $h(p q) \equiv 2(\bmod 4)$.

Les cas 3, 4 et 6 sont à rejeter car l'extension $K / \mathbb{Q}\left(\sqrt{d_{1} d_{2}}\right)$ est supposée ramifiée.

Le cas 5 est aussi à rejeter car, d'après le théorème 3, le 2-groupe de classes de $K$ est trivial ou cyclique, ce qui est contraire aux hypothèses. Ainsi ce qui reste sont les cas 1 et 2 .

Afin d'avoir $K / \mathbb{Q}\left(\sqrt{d_{1} d_{2}}\right)$ ramifiée, $K$ doit prendre l'une des formes suivantes: Dans le cas 1:

$$
[K=\mathbb{Q}(\sqrt{2}, \sqrt{p q}) \text { ou bien } K=\mathbb{Q}(\sqrt{q}, \sqrt{2 p})] \text { et }\left[\left(\frac{2}{p}\right)=-1 \text { ou }\left(\frac{q}{p}\right)=-1\right] \text {. }
$$

Dans le cas 2:

$$
\left[K=\mathbb{Q}\left(\sqrt{q}, \sqrt{p q^{\prime}}\right) \text { ou bien } K=\mathbb{Q}\left(\sqrt{q^{\prime}}, \sqrt{p q}\right)\right] \text { et }\left[\left(\frac{q}{p}\right)=-1 \text { ou }\left(\frac{q^{\prime}}{p}\right)=-1\right] \text {. }
$$

Théorème 9. Soient $K=\mathbb{Q}\left(\sqrt{d_{1}}, \sqrt{d_{2}}\right)$ où $d_{1}$ et $d_{2}$ sont deux entiers naturels premiers entre eux. On suppose que l'extension $K / \mathbb{Q}\left(\sqrt{d_{1} d_{2}}\right)$ est ramifiée et que $\left[K^{(*)}: K\right]=2$; alors le 2-groupe de classes de $K$ est de type $(2,2)$ si et seulement si l'un des deux cas suivants est vérifié:

1) $d_{1}=2, d_{2}=p q$ où $p$ et $q$ sont deux premiers tels que $p \equiv-q \equiv 1(\bmod 4)$ et $\left(\frac{2}{p}\right)=-\left(\frac{q}{p}\right)=1$.

2) $d_{1}=q, d_{2}=p q^{\prime}$ où $p, q$ et $q^{\prime}$ sont des premiers tels que $p \equiv-q \equiv-q^{\prime} \equiv$ $1(\bmod 4)$ et $\left(\frac{2}{p}\right)=\left(\frac{q}{p}\right)=-\left(\frac{q^{\prime}}{p}\right)=1$.

Preuve. 1) D'après le théorème 2, le 2-groupe de classes de $K$ est de rang 2 si et seulement si $\left(\frac{2}{p}\right)=1$. Alors, dans ce qui suit, on suppose que c'est le cas. On sait d'après ce qui précède que $\left(\frac{2}{p}\right)=-1$ ou $\left(\frac{q}{p}\right)=-1$; par suite $\left(\frac{q}{p}\right)=-1$, et alors, d'après [Kaplan 1976], on a $h(p q) \equiv h(2 p q) \equiv 2(\bmod 4)$. D'autre part, d'après [Kubota 1956], on a $Q_{K} \in\{1,2,4\}$ et comme $\operatorname{rang}\left(C_{2, K}\right)=2$, alors $4 \mid h(K)$ et par suite $Q_{K}=4$ et $h(K)=4$.

2) Soit $K=\mathbb{Q}\left(\sqrt{q}, \sqrt{p q^{\prime}}\right)$. D'après le théorème 3, le 2-groupe de classes de $K$ est de rang égal à 2 si et seulement si $\left(\frac{2}{p}\right)=\left(\frac{q}{p}\right)=1$. On suppose dans ce qui suit que c'est le cas. On sait d'après ce qui précède que $\left(\frac{q}{p}\right)=-1$ ou $\left(\frac{q^{\prime}}{p}\right)=-1$; par suite $\left(\frac{q^{\prime}}{p}\right)=-1$, et alors, d'après [Kaplan 1976], on a $h(p q) \equiv h(2 p q) \equiv 2(\bmod 4)$. 
D'autre part d'après [Kubota 1956], on a $Q_{K} \in\{1,2,4\}$ et comme $\operatorname{rang}\left(C_{2, K}\right)=2$, alors $4 \mid h(K)$ et par suite $Q_{K}=4$ et $h(K)=4$. Ainsi le théorème est démontré.

Dans la suite on va étudier le problème de capitulation des 2-classes d'idéaux de $K$ dans les deux cas du théorème 9 .

Étude de la capitulation dans le cas 1 du théorème 9. Dans ce cas, on a $K=$ $\mathbb{Q}(\sqrt{2}, \sqrt{p q})$ où $p$ et $q$ sont deux premiers tels que $p \equiv-q \equiv 1(\bmod 4)$ et $\left(\frac{2}{p}\right)=$ $-\left(\frac{q}{p}\right)=1$ et $K^{(*)}=\mathbb{Q}(\sqrt{2}, \sqrt{p}, \sqrt{q})$.

Proposition 1. Soient $p, q$ deux premiers tels que $p \equiv-1(\bmod 4)$ et que $\left(\frac{2}{p}\right)=$ $-\left(\frac{q}{p}\right)=1$. Alors $K_{2}^{(1)} \neq K_{2}^{(2)}$ si et seulement si $\left(\frac{2}{p}\right)_{4}=(-1)^{(p-1) / 8}=1$.

Preuve. Soit $L=\mathbb{Q}(\sqrt{q}, \sqrt{2 p})$; alors d'après le théorème 3, le 2-groupe de classes de $L$ est cyclique. De plus, l'extension $K^{(*)} / L$ est non ramifiée; par suite $K^{(*)}$ et $L$ ont le même 2-corps de classes de Hilbert qui est $K_{2}^{(2)}$. Ainsi $h\left(K_{2}^{(1)}\right)=\frac{1}{2} h\left(K^{(*)}\right)=$ $\frac{1}{4} h(L)$ et par conséquent on a $K_{2}^{(1)} \neq K_{2}^{(2)}$ si et seulement si $8 \mid h(L)$. Donc on est ramené à étudier la 2-partie du nombre de classes de $L$. D'après [Kaplan 1976], on a $h(2 p q) \equiv 2(\bmod 4)$ et d'après la théorie des genres $h(q)=1$ et $2 \mid h(2 p)$. On suppose que $\left(\frac{2}{p}\right)_{4} \neq(-1)^{(p-1) / 8}$; alors d'après [Kučera 1995], $h(2 p) \equiv 2(\bmod 4)$ et $e(2 p)=1$. D'autre part, d'après le lemme 4 , on a $Q_{L}=4$. Ainsi on a $h(L) \equiv$ $4(\bmod 8)$ et donc $K_{2}^{(1)}=K_{2}^{(2)}$. On suppose que $\left(\frac{2}{p}\right)_{4}=(-1)^{(p-1) / 8}$; alors, d'après [Kučera 1995], on a $4 \mid h(2 p)$. On distingue deux cas:

- On suppose que $\left(\frac{2}{p}\right)_{4}=(-1)^{(p-1) / 8}=1$; alors d'après [Kučera 1995], on a:

Si $e(2 p)=-1$, alors $8 \mid h(2 p)$ et d'après le lemme 4 , on a $Q_{L}=2$ Par suite $Q_{L}=2$ et par conséquent $8 \mid h(L)$. Si $e(2 p)=1$, alors d'après le lemme 4 , on a $Q_{L}=4$ et par conséquent $8 \mid h(L)$.

- On suppose que $\left(\frac{2}{p}\right)_{4}=(-1)^{(p-1) / 8}=-1$; alors d'après [Kučera 1995], on a $h(2 p) \equiv 4(\bmod 8)$ et $e(2 p)=-1$. D'après le lemme $4, Q_{L}=2$. Par suite, on a $h(L) \equiv 4(\bmod 8)$, d'où $K_{2}^{(1)}=K_{2}^{(2)}$. Ainsi la proposition est démontrèe.

Dans la suite, on va déterminer les classes engendrant le 2-groupe de classes de $K$. On note par $O_{M}$ l'anneau des entiers d'un corps $M$.

Comme $\left(\frac{2}{p}\right)=1$, alors $p O_{\mathbb{Q}(\sqrt{2})}=\mathscr{P}_{1} \mathscr{P}_{2}$ où $\mathscr{P}_{1}$ et $\mathscr{P}_{2}$ sont deux idéaux premiers différents de $\mathbb{Q}(\sqrt{2})$. De plus pour $i \in\{1,2\}$ on a $\mathscr{P}_{i} O_{K}=Y_{i}^{2}$ où $Y_{i}$ est un idéal premier de $K$.

Proposition 2. Soient $p, q$ deux premiers tels que $p \equiv-1(\bmod 4)$ et que $\left(\frac{2}{p}\right)=$ $-\left(\frac{q}{p}\right)=1$. On suppose que $\left(\frac{2}{p}\right)_{4}=(-1)^{(p-1) / 8}=1 ;$ alors le 2-groupe de classes de $K$ est engendré par les classes d'idéaux de $Y_{1}$ et de $Y_{2}$.

Preuve. On sait que le nombre de classes de $\mathbb{Q}(\sqrt{2})$ est égal à 1 . Comme $\mathscr{P}_{1}$ et $\mathscr{P}_{2}$ sont deux idéaux premiers de $\mathbb{Q}(\sqrt{2})$, ils sont principaux. Or pour $i \in\{1,2\}$ on a $\mathscr{P}_{i} O_{K}=Y_{i}^{2}$, donc les classes $\left[Y_{1}\right]$ et $\left[Y_{2}\right]$ sont des 2 -classes de $K$. Montrons que $Y_{1}$ n'est pas principal. 
On vérifie facilement que $Y_{1}$ reste inerte dans $K^{(*)}$; alors d'après la loi de réciprocité d'Artin appliquée à l'extension $K^{(*)} / K$, l'idéal $Y_{1}$ n'est pas principal. Montrons que $Y_{1} Y_{2}$ n'est pas principal. On a $\mathcal{N}_{K / \mathbb{Q}(\sqrt{2})}\left(Y_{1} Y_{2}\right)=\mathscr{P}_{1} \mathscr{P}_{2}=p O_{\mathbb{Q}(\sqrt{2})}$. On suppose que $Y_{1} Y_{2}$ est principal; alors il existe une unité $u$ de $\mathbb{Q}(\sqrt{2})$ tel que $p u$ est norme dans l'extension $K / \mathbb{Q}(\sqrt{2})$, ce qui est impossible. En effet:

En utilisant le symbole du reste normique, on sait que $p u$ est norme dans cette extension si et seulement si $\left(\frac{p u, p q}{2}\right)=1$ pour tout premier 2 de $\mathbb{Q}(\sqrt{2})$ ramifié dans $K$ (voir [Azizi et Mouhib 2001]).

D'autre part, l'idéal premier $\mathscr{P}_{1}$ de $\mathbb{Q}(\sqrt{2})$ se ramifie dans $K$ et on a

$$
\left(\frac{-1, p q}{\mathscr{P}_{1}}\right)=\left(\frac{-1, p}{\mathscr{P}_{1}}\right)=\left(\frac{-1}{p}\right)=1 \quad \text { et } \quad\left(\frac{\varepsilon_{2}, p q}{\mathscr{P}_{1}}\right)=\left(\frac{\varepsilon_{2}, p}{\mathscr{P}_{1}}\right)\left(\frac{\varepsilon_{2}, q}{\mathscr{P}_{1}}\right)=\left(\frac{\varepsilon_{2}, p}{\mathscr{P}_{1}}\right) .
$$

Or, $\left(\frac{\varepsilon_{2}, p}{\mathscr{P}_{1}}\right)=\left(\frac{2}{p}\right)_{4}(-1)^{(p-1) / 8}$ d'après [Azizi et Mouhib 2001]. Comme $\left(\frac{2}{p}\right)_{4}=$ $(-1)^{(p-1) / 8}=1$, on a $\left(\frac{\varepsilon_{2}, p}{\mathscr{P}_{1}}\right)=1$ et donc $\left(\frac{\varepsilon_{2}, p q}{\mathscr{P}_{1}}\right)=1$. Ainsi l'unité $u$ de $\mathbb{Q}(\sqrt{2})$ vérifie $\left(\frac{u, p q}{\mathscr{P}_{1}}\right)=1$. De plus, on a

$$
\left(\frac{p, p q}{\mathscr{P}_{1}}\right)=\left(\frac{p, p}{\mathscr{P}_{1}}\right)\left(\frac{p, q}{\mathscr{P}_{1}}\right)=\left(\frac{q}{p}\right)=-1 .
$$

D'où $\left(\frac{p u, p q}{\mathscr{P}_{1}}\right)=-1$, par conséquent on a une contradiction. Ainsi $Y_{1} Y_{2}$ n'est pas principal et la proposition est démontrée.

Dans la suite, on va déterminer les 2-classes de $K$ qui capitulent dans $K^{(*)}$.

On sait que $Y_{1}$ et $Y_{2}$ restent inerte dans $K^{(*)}$, donc $Y_{1} O_{K^{(*)}}=Q_{1}$ et $Y_{2} O_{K^{(*)}}=Q_{2}$ où $2_{1}$ et $2_{2}$ sont deux idéaux premiers de $K^{(*)}$.

D'autre part on a $p O_{L}=2^{2}$ où $L=\mathbb{Q}(\sqrt{q}, \sqrt{2 p})$ et 2 est un idéal premier de $L$; par suite $2 O_{K^{(*)}}=Y_{1} Y_{2}$.

Proposition 3. En gardant les mêmes notations, les idéaux premiers $\mathscr{2}_{1}$ et $\mathscr{2}_{2}$ sont principaux si et seulement si l'idéal premier 2 est principal.

Preuve. On sait que si $\mathscr{2}_{1}$ est principal; alors $2=\mathcal{N}_{K^{(*) / L}}\left(2_{1}\right)$ est principal.

Inversement, si l'idéal premier 2 est principal, on raisonne comme suit:

On sait que le 2-groupe de classes de $L$ est cyclique et que $K^{(*)}$ et $L$ ont le même 2-corps de classes de Hilbert qui est $K_{2}^{(2)}$. D'autre part, d'après la théorie des corps de classes de Hilbert, le noyau de l'application d'Artin dans l'extension $K_{2}^{(2)} / L$ est réduit au groupe des idéaux fractionnaires principaux de $L$. Comme l'idéal premier 2 est principal, 2 se décompose complètement dans $K_{2}^{(2)}$; par suite les idéaux premiers $2_{1}$ et $2_{2}$ se décomposent complètement dans $K_{2}^{(2)}$. Comme $K_{2}^{(2)}$ est le 2-corps de classes de Hilbert de $K^{(*)}$, le noyau de l'application d'Artin dans l'extension $K_{2}^{(2)} / K^{(*)}$ est réduit au groupe des idéaux fractionnaires principaux de $K^{(*)}$. Ainsi $2_{1}$ et $2_{2}$ sont principaux et la proposition est démontrée.

Théorème 10. Soient $p$ et $q$ deux premiers tels que $p \equiv-q \equiv 1(\bmod 4),\left(\frac{2}{p}\right)=$ $-\left(\frac{q}{p}\right)=1$ et $K=\mathbb{Q}(\sqrt{2}, \sqrt{p q})$. On suppose que $\left(\frac{2}{p}\right)_{4}=(-1)^{(p-1) / 8}=1 ;$ alors toutes les 2 -classes de $K$ capitulent dans $K^{(*)}$ et le groupe $G=\operatorname{Gal}\left(K_{2}^{(2)} / K\right)$ est diédral. 
Preuve. On sait que le 2-groupe de classes de $K$ est engendré par les classes d'idéaux $\left[Y_{1}\right]$ et $\left[Y_{2}\right]$ définis dans la proposition 2. Montrons que $Y_{1} Y_{2}$ capitule dans $K^{(*)}$.

On a $p O_{\mathbb{Q}(\sqrt{2 p q})}=\mathscr{P}^{2}$ où $\mathscr{P}$ est un idéal premier de $\mathbb{Q}(\sqrt{2 p q})$; par suite $\mathscr{P} O_{K}=$ $Y_{1} Y_{2}$. Ainsi, il existe deux 2-classes ambigues de $K$ relativement à $\mathbb{Q}(\sqrt{2 p q})$, la classe triviale et la classe $\left[Y_{1} Y_{2}\right]$. Comme $K^{(*)} / \mathbb{Q}(\sqrt{2 p q})$ est une extension abélienne et $K^{(*)} / K$ est une extension non ramifiée, $K^{(*)}$ est le 2-corps de genres relatif à l'extension $K / \mathbb{Q}(\sqrt{2 p q})$. On sait, d'après [Terada 1971], que toutes les 2-classes ambigues de $K^{(*)} / \mathbb{Q}(\sqrt{2 p q})$ capitulent dans $K^{(*)}$. Ainsi la classe $\left[Y_{1} Y_{2}\right]$ capitule dans $K^{(*)}$. Montrons que $Y_{1}$ capitule dans $K^{(*)}$.

On sait que $Y_{1} O_{K^{(*)}}=2_{1}$ où $2_{1}$ est un idéal premier de $K^{(*)}$ et que $p O_{L}=2^{2}$ où 2 est un idéal premier de $L$. D'après la proposition $3, Y_{1}$ capitule dans $K^{(*)}$ si et seulement si 2 est principal. Montrons que 2 est principal.

Soit $\varepsilon_{q}=x+y \sqrt{q}$ l'unité fondamentale de $\mathbb{Q}(\sqrt{q})$. Alors, d'après [Azizi 2000], $x \pm 1$ est un carré dans $\mathbb{N}$. Donc il existe deux entiers naturels $y_{1}$ et $y_{2}$ tels que $x \pm 1=y_{1}^{2}, x \mp 1=y_{2}^{2}$ et $y_{1} y_{2}=y$; par suite $\sqrt{\varepsilon_{q}}=\frac{1}{2} \sqrt{2} y_{1}+\frac{1}{2} \sqrt{2 q} y_{2}$. De plus, on a $p O_{L}=\left(\sqrt{p} \sqrt{\varepsilon_{q}}\right)^{2} \varepsilon_{q}^{-1} O_{L}=2^{2}$. Comme $\sqrt{p} \sqrt{\varepsilon}$ est un entier de $L$, l'idéal 2 est principal; par suite l'idéal $2_{1}$ est principal. Ainsi, toutes les 2-classes de $K$ capitulent dans $K^{(*)}$. Comme $\left(\frac{2}{p}\right)_{4}=(-1)^{(p-1) / 8}=1$, alors $K_{2}^{(1)} \neq K_{2}^{(2)}$ et d'après [Kisilevsky 1976], le groupe $\operatorname{Gal}\left(K_{2}^{(2)} / K\right)$ est diédral.

Étude de la capitulation dans le cas 2 du théorème 9. Ce cas est similaire au cas 1 , et on suit les mêmes étapes.

Dans le cas 2 du théorème 9 , on a $K=\mathbb{Q}\left(\sqrt{q}, \sqrt{p q^{\prime}}\right)$ où $p, q$ et $q^{\prime}$ sont des premiers tels que $p \equiv-q \equiv-q^{\prime} \equiv 1(\bmod 4)$ et $\left(\frac{2}{p}\right)=\left(\frac{q}{p}\right)=-\left(\frac{q^{\prime}}{p}\right)=1$ et $K^{(*)}=\mathbb{Q}\left(\sqrt{q}, \sqrt{p}, \sqrt{q^{\prime}}\right)$.

Proposition 4. Soient $p, q$, $q^{\prime}$ des premiers différents tels que $p \equiv-q \equiv-q^{\prime} \equiv$ $1(\bmod 4),\left(\frac{2}{p}\right)=\left(\frac{q}{p}\right)=-\left(\frac{q^{\prime}}{p}\right)=1$ et $K=\mathbb{Q}\left(\sqrt{q}, \sqrt{p q^{\prime}}\right)$. Alors $K_{2}^{(1)} \neq K_{2}^{(2)}$ si et seulement si $8 \mid h(p q)$.

Preuve. D'après le théorème 3 , le 2-groupe de classes de $L=\mathbb{Q}\left(\sqrt{q^{\prime}}, \sqrt{p q}\right)$ est cyclique. De plus, l'extension $K^{(*)} / F$ est non ramifiée, donc $K^{(*)}$ et $L$ ont le même 2-corps de classes de Hilbert qui est $K_{2}^{(2)}$. Alors comme dans le cas 1 du théorème 9 , on a $K_{2}^{(1)} \neq K_{2}^{(2)}$ si et seulement si $8 \mid h(L)$. On est donc ramené à étudier la 2-partie du nombre de classes de $L$. D'après [Kaplan 1976] on a $h\left(q p q^{\prime}\right)=2$ et $4 \mid h(q p)$. D'après le lemme 5 , on a $Q_{L}=2$.

Si $h(p q) \equiv 4(\bmod 8)$, on a $h(L) \equiv 4(\bmod 8)$; par suite $K_{2}^{(1)}=K_{2}^{(2)}$. Si $8 \mid h(p q)$, on a $8 \mid h(L)$; par suite $K_{2}^{(1)} \neq K_{2}^{(2)}$. D'où la proposition est démontrée.

Dans la suite on va déterminer les classes engendrant le 2-groupe de classes de $K$. Comme $\left(\frac{q}{p}\right)=1$, l'idéal premier $p$ se décompose dans $\mathbb{Q}(\sqrt{q})$ et on a $p O_{\mathbb{Q}(\sqrt{q})}=\mathscr{P}_{1} \mathscr{P}_{2}$ où $\mathscr{P}_{1}$ et $\mathscr{P}_{2}$ sont deux idéaux premiers différents de $\mathbb{Q}(\sqrt{q})$. Comme $p$ est ramifié dans $K$, alors $\mathscr{P}_{i} O_{K}=Y_{i}^{2}$ où $Y_{i}$ est un idéal premier de $K$. 
Proposition 5. Soient $p, q, q^{\prime}$ des premiers différents tels que $p \equiv-q \equiv-q^{\prime} \equiv 1$ $(\bmod 4),\left(\frac{2}{p}\right)=\left(\frac{q}{p}\right)=-\left(\frac{q^{\prime}}{p}\right)=1$ et $K=\mathbb{Q}\left(\sqrt{q}, \sqrt{p q^{\prime}}\right)$. Alors le 2-groupe de classes de $K$ est engendré par $\left[Y_{1}^{l}\right]$ et $\left[Y_{2}^{l}\right]$ où l est le nombre de classes de $\mathbb{Q}(\sqrt{q})$.

Preuve. Le corps quadratique $\mathbb{Q}(\sqrt{q})$ est de nombre de classes impair. Soit $l$ le nombre de classes de $\mathbb{Q}(\sqrt{q})$; alors $\mathscr{P}_{1}^{l}$ et $\mathscr{P}_{2}^{l}$ sont des idéaux principaux de $\mathbb{Q}(\sqrt{q})$. Par suite $\left[Y_{1}^{l}\right]$ et $\left[Y_{2}^{l}\right]$ sont des 2 -classes de $K$. Les idéaux premiers $Y_{1}$ et $Y_{2}$ ne sont pas principaux. En effet:

On vérifie que $Y_{1}$ et $Y_{2}$ restent inerte dans $K^{(*)}$. Alors d'après la loi de réciprocité d'Artin appliquée à l'extension $K^{(*)} / K$, les idéaux premiers $Y_{1}$ et $Y_{2}$ ne sont pas principaux. Comme $l$ est impair et l'extension $K^{(*)} / K$ est de degré 2, alors $Y_{1}^{l}$ et $Y_{2}^{l}$ ne sont pas principaux. Montrons que $Y_{1}^{l} Y_{2}^{l}$ est non principal.

On a $\mathcal{N}_{K / \mathbb{Q}(\sqrt{q})}\left(Y_{1}^{l} Y_{2}^{l}\right)=\mathscr{P}_{1}^{l} \mathscr{P}_{2}^{l}=p^{l} O_{\mathbb{Q}(\sqrt{q})}$. On suppose que $Y_{1}^{l} Y_{2}^{l}$ est principal; alors il existe une unité $u$ de $\mathbb{Q}(\sqrt{q})$ tel que $p^{l} u$ est norme dans l'extension $K / \mathbb{Q}(\sqrt{q})$. Ce qui est impossible. En effet:

Comme dans le cas 1, en utilisant le symbole du reste normique, on a:

l'idéal $\mathscr{P}_{1}$ est un premier de $\mathbb{Q}(\sqrt{q})$ ramifié dans $K$. On a $\left(\frac{-1, p q^{\prime}}{\mathscr{P}_{1}}\right)=\left(\frac{-1, p}{\mathscr{P}_{1}}\right)=$ $\left(\frac{-1}{p}\right)=1$ et $\left(\frac{\varepsilon_{q}, p q^{\prime}}{\mathscr{P}_{1}}\right)=\left(\frac{\varepsilon_{q}, p}{\mathscr{P}_{1}}\right)$. D'autre part, d'après la preuve du théorème 10 , il existe deux nombres rationnels $y_{1}$ et $y_{2}$ tels que $\sqrt{\varepsilon_{q}}=y_{1} \sqrt{2}+y_{2} \sqrt{2 q}$; par suite $\varepsilon_{q}=2 v^{2}$ où $v$ est un élément de $\mathbb{Q}(\sqrt{q})$ et on a $\left(\frac{\varepsilon_{q}, p}{\mathscr{P}_{1}}\right)=\left(\frac{2, p}{\mathscr{P}_{1}}\right)=\left(\frac{2}{p}\right)=1$. Ainsi $\left(\frac{u, p q^{\prime}}{\mathscr{P}_{1}}\right)=1$ et de plus

$$
\left(\frac{p^{l}, p q^{\prime}}{\mathscr{P}_{1}}\right)=\left(\frac{p^{l}, p}{\mathscr{P}_{1}}\right)\left(\frac{p^{l}, q^{\prime}}{\mathscr{P}_{1}}\right)=\left(\frac{q^{\prime}}{p}\right)^{l}=-1 .
$$

Donc on a une contradiction. Par conséquent la proposition est démontrée.

Dans la suite on va déterminer les 2-classes de $K$ qui capitulent dans $K^{(*)}$.

On sait que le 2-groupe de classes de $K=\mathbb{Q}\left(\sqrt{q}, \sqrt{p q^{\prime}}\right)$ est engendré par $\left[Y_{1}^{l}\right]$ et $\left[Y_{2}^{l}\right]$ où $l$ est le nombre de classes de $\mathbb{Q}(\sqrt{q})$. On pose $Y_{1} O_{K^{*}}=\mathscr{2}_{1}$ et $Y_{2} O_{K^{*}}=2_{2}$ où $2_{1}$ et $\mathscr{2}_{2}$ sont deux idéaux premiers de $K^{(*)}$. D'autre part, on a $q O_{L}=2^{2}$ où $L=\mathbb{Q}\left(\sqrt{q^{\prime}}, \sqrt{p q}\right)$ et 2 est un idéal premier de $L$.

Proposition 6. En gardant les mêmes notations, 2 est principal si et seulement si $2_{1}$ et $2_{1}$ sont principaux.

Preuve. Le corps $L=\mathbb{Q}\left(\sqrt{q^{\prime}}, \sqrt{p q}\right)$ est de 2-groupe de classes cyclique, alors la preuve de la proposition 3 reste valable ici.

Théorème 11. Soient $p, q$ et $q^{\prime}$ des premiers tels que $p \equiv-q \equiv-q^{\prime} \equiv 1(\bmod 4)$, $\left(\frac{2}{p}\right)=\left(\frac{q}{p}\right)=-\left(\frac{q^{\prime}}{p}\right)=1$ et $K=\mathbb{Q}\left(\sqrt{q}, \sqrt{p q^{\prime}}\right)$. On suppose que $8 \mid h\left(p q^{\prime}\right)$; alors toutes les 2-classes de $K$ capitulent dans $K^{(*)}$ et le groupe $G=\operatorname{Gal}\left(K_{2}^{(2)} / K\right)$ est diédral.

Preuve. On sait, d'après la proposition 5, que le 2-groupe de classes de $K$ est engendré par les classes $\left[Y_{1}^{l}\right]$ et $\left[Y_{1}^{l}\right]$. Montrons que $Y_{1}^{l} Y_{2}^{l}$ capitule dans $K^{(*)}$. Comme dans le cas 1 du théorème 9 , on trouve que $\left[Y_{1}^{l} Y_{2}^{l}\right]$ est la seule 2-classe ambigue non 
triviale relativement à l'extension $K / \mathbb{Q}\left(\sqrt{p q q^{\prime}}\right)$ et $K^{(*)}$ est le 2-corps de genres relatif de l'extension $K / \mathbb{Q}\left(\sqrt{p q q^{\prime}}\right)$. D'après [Terada 1971], $Y_{1}^{l} Y_{2}^{l}$ capitule dans $K^{(*)}$. Montrons que $Y_{1}$ capitule dans $K^{(*)}$.

On sait que $Y_{1} O_{K^{(*)}}=2_{1}$ où $2_{1}$ est un idéal premier de $K^{(*)}$ et que $p O_{L}=2^{2}$ où 2 est un idéal premier de $L$. D'après la proposition $6, Y_{1}$ capitule dans $K^{(*)}$ si et seulement si 2 est principal. Montrons que 2 est principal.

Soit $\varepsilon_{p q q^{\prime}}$ l'unité fondamentale de $\mathbb{Q}\left(\sqrt{p q q^{\prime}}\right)$. Comme $\left(\frac{2}{p}\right)=\left(\frac{q}{p}\right)=-\left(\frac{q^{\prime}}{p}\right)=1$, il existe, d'après le lemme 5 , deux entiers naturels $y_{1}$ et $y_{2}$ tels que $\sqrt{\frac{p}{\varepsilon_{p q q^{\prime}}}}=$ $\frac{1}{2} y_{1} \sqrt{q}+\frac{1}{2} y_{2} \sqrt{p q^{\prime}}$. Par conséquent on a

$$
p O_{L}=\left(\sqrt{p} \sqrt{\varepsilon_{p q q^{\prime}}}\right)^{2} \varepsilon_{p q q^{\prime}}^{-1} O_{L}=2^{2} .
$$

Comme $\sqrt{p} \sqrt{\varepsilon_{p q q^{\prime}}}$ est un entier de $L$, l'idéal 2 est principal. Par suite $2_{1}^{l}$ et $2_{2}^{l}$ sont principaux. Ainsi, toutes les 2-classes de $K$ capitulent dans $K^{(*)}$. De plus, comme $8 \mid h(L)$, alors $K_{2}^{(1)} \neq K_{2}^{(2)}$ et d'après [Kisilevsky 1976], le groupe $\operatorname{Gal}\left(K_{2}^{(2)} / K\right)$ est diédral.

Conclusion. Soient $d_{1}$ et $d_{2}$ deux entiers naturels sans facteurs carrés et premiers entre eux, $K=Q\left(\sqrt{d_{1} d_{2}}\right), K_{2}^{(1)}$ le 2-corps de classes de Hilbert de $K$ et $K_{2}^{(2)}$ le 2corps de classes de Hilbert de $K_{2}^{(1)}$. On suppose que $\operatorname{Gal}\left(K_{2}^{(1)} / K\right) \simeq \mathbb{Z} / 2 \mathbb{Z} \times \mathbb{Z} / 2 \mathbb{Z}$, $K^{(*)} \neq K_{2}^{(1)}$ et l'extension $K / Q\left(\sqrt{d_{1} d_{2}}\right)$ est ramifiée. Alors il existe toujours une sous-extension propre de $K_{2}^{(1)} / K$ où toutes les 2-classes de $K$ capitulent. Plus précisément le groupe $\operatorname{Gal}\left(K_{2}^{(2)} / K\right)$ est abélien ou diédral.

Exemples numériques. Soit $K=\mathbb{Q}\left(\sqrt{d_{1}}, \sqrt{d_{2}}\right)$ où $d_{1}$ et $d_{2}$ sont deux entiers naturels sans facteurs carrés et premiers entre eux tels que $K / \mathbb{Q}\left(\sqrt{d_{1} d_{2}}\right)$ est ramifiée. On suppose que $\operatorname{Gal}\left(K_{2}^{(1)} / K\right) \simeq \mathbb{Z} / 2 \mathbb{Z} \times \mathbb{Z} / 2 \mathbb{Z}$ et $K^{(*)} \neq K_{2}^{(1)}$. Soit alors $L$ le corps biquadratique contenu dans $K^{(*)}$ tel que le 2-groupe de classes de $L$ est cyclique et l'extension $K^{(*)} / L$ est non ramifiée.

On sait, d'après le théorème 9, qu'il existe deux cas de corps $K$ dont le 2-groupe de classes est de type $(2,2)$. On donne des exemples numériques corréspondant à chaque cas. Dans la suite $p, q, q^{\prime}$ sont des premiers différents vérifiant $p \equiv-q \equiv$ $-q^{\prime} \equiv 1(\bmod 4)$.

Cas 1) $\boldsymbol{d}_{\mathbf{1}}=\mathbf{2}$ et $\boldsymbol{d}_{\mathbf{2}}=p q$ où $\left(\frac{\mathbf{2}}{p}\right)=-\left(\frac{q}{p}\right)=\mathbf{1}$.

- Soient $p=113$ et $q=3$. On a $\left(\frac{2}{p}\right)=1,\left(\frac{p}{q}\right)=-1,\left(\frac{2}{p}\right)_{4}=(-1)^{(p-1) / 8}=1$ et $h(2 p)=8$. De plus on a $L=\mathbb{Q}(\sqrt{q}, \sqrt{2 p})$ et $e(2 p)=-1$; par suite $Q_{L}=2$. Ainsi d'après la proposition 1 , on a $K_{2}^{(2)} \neq K_{2}^{(1)}$. D'autre part on a

$$
\left|\operatorname{Gal}\left(K_{2}^{(2)} / K\right)\right|=\left[K_{2}^{(2)}: L\right]=h(L)=\frac{Q_{L} h(2 p) h(2 q p) h(q)}{4}=h(2 p)=8
$$

et d'après le théorème 10 , le groupe $\operatorname{Gal}\left(K_{2}^{(2)} / K\right)$ est diédral d'ordre 8 . 
- Soient $p=3313$ et $q=11$. On a $\left(\frac{2}{p}\right)=1,\left(\frac{p}{q}\right)=-1$,

$$
\left(\frac{2}{p}\right)_{4}=(-1)^{(p-1) / 8}=1 \quad \text { et } \quad h(2 p)=16 .
$$

De plus on a $L=\mathbb{Q}(\sqrt{q}, \sqrt{2 p})$ et $e(2 p)=-1$; par suite $Q_{L}=2$ (voir lemme 4). Ainsi d'après la proposition 1 , on a $K_{2}^{(2)} \neq K_{2}^{(1)}$. D'autre part on a

$$
\left|\operatorname{Gal}\left(K_{2}^{(2)} / K\right)\right|=h(L)=h(2 p)=16
$$

et d'après le théorème 10 , le groupe $\operatorname{Gal}\left(K_{2}^{(2)} / K\right)$ est diédral d'ordre 16 .

- Soient $p=41$ et $q=19$. On a $\left(\frac{2}{p}\right)=1$ et $\left(\frac{q}{p}\right)=-1$; de plus

$$
\left(\frac{2}{p}\right)_{4}=(-1)^{(p-1) / 8}=-1 \text {. }
$$

Alors, d'après la proposition $1, K_{2}^{(1)}=K_{2}^{(2)}$ et le groupe $\operatorname{Gal}\left(K_{2}^{(2)} / K\right)$ est abélien.

Cas 2) $d_{\mathbf{1}}=q$ et $\boldsymbol{d}_{\mathbf{2}}=p q^{\prime}$ où $\left(\frac{2}{p}\right)=\left(\frac{q}{p}\right)=-\left(\frac{q^{\prime}}{p}\right)=\mathbf{1}$.

- Soient $p=433, q=3$ et $q^{\prime}=7$. On a

$$
\left(\frac{2}{p}\right)=\left(\frac{q}{p}\right)=-\left(\frac{q^{\prime}}{p}\right)=1 \quad \text { et } \quad h(p q)=8 .
$$

Ainsi, d'après la proposition 4 , on a $K_{2}^{(1)} \neq K_{2}^{(2)}$. D'autre part, on a $L=$ $\mathbb{Q}\left(\sqrt{q^{\prime}}, \sqrt{q p}\right), Q_{L}=2$ et

$$
\left|\operatorname{Gal}\left(K_{2}^{(2)} / K\right)\right|=h(L)=\frac{Q_{L} h(q p) h\left(q q^{\prime} p\right) h\left(q^{\prime}\right)}{4}=h(q p)=8 .
$$

D'après le théorème 11 , le groupe $\operatorname{Gal}\left(K_{2}^{(2)} / K\right)$ est diédral d'ordre 8 .

- Soient $p=17, q=7$ et $q^{\prime}=31$. On a

$$
\left(\frac{2}{p}\right)=\left(\frac{q}{p}\right)=-\left(\frac{q^{\prime}}{p}\right)=1 \quad \text { et } \quad\left(\frac{2}{p}\right)_{4} \neq(-1)^{(p-1) / 8} .
$$

D'après la proposition $4, \operatorname{Gal}\left(K_{2}^{(2)} / K\right)$ est abélien.

- Soient $p=1009, q^{\prime}=11$ et $q=7$. On a

$$
\left(\frac{2}{p}\right)=\left(\frac{q}{p}\right)=-\left(\frac{q^{\prime}}{p}\right)=1 \quad \text { et } \quad h(p q)=8 .
$$

D'après la proposition $4, K_{2}^{(1)} \neq K_{2}^{(2)}$. D'après le théorème $11, \operatorname{Gal}\left(K_{2}^{(2)} / K\right)$ est diédral d'ordre 8.

\section{References}

[Azizi 2000] A. Azizi, "Sur la capitulation des 2-classes d'idéaux de $\mathbf{k}=\mathbf{Q}(\sqrt{2 p q}, i)$ où $p \equiv-q \equiv$ $1 \bmod 4$ ", Acta Arith. 94:4 (2000), 383-399. MR 2001k:11221 Zbl 0953.11033

[Azizi et Mouhib 2001] A. Azizi et A. Mouhib, "Sur le rang du 2-groupe de classes de $\mathbf{Q}(\sqrt{m}, \sqrt{d})$ où $m=2$ ou un premier $p \equiv 1$ (mod 4)", Trans. Amer. Math. Soc. 353:7 (2001), 2741-2752. MR 2002b:11152

[Azizi et Mouhib $\geq 2008$ ] A. Azizi et A. Mouhib, "Sur le 2-groupe de classes du corps de genres de certains corps biquadratiques", Ann. Sci. Math. Québec. To appear.

[Benjamin et Snyder 1995] E. Benjamin et C. Snyder, "Real quadratic number fields with 2-class group of type (2, 2)”, Math. Scand. 76:2 (1995), 161-178. MR 96m:11094 Zbl 0847.11058 
[Benjamin et al. 1998] E. Benjamin, F. Lemmermeyer et C. Snyder, "Real quadratic fields with abelian 2-class field tower", Journal of Number Theory 73:2 (1998), 182-194. MR 2000c:11179 Zbl 0919.11073

[Cohn 1978] H. Cohn, A classical invitation to algebraic numbers and class fields, Springer-Verlag, New York, 1978. MR 80c:12001

[Couture et Derhem 1992] R. Couture et A. Derhem, "Un problème de capitulation", C. R. Acad. Sci. Paris Sér. I Math. 314:11 (1992), 785-788. MR 93c:11100 Zbl 0778.11059

[Gorenstein 1980] D. Gorenstein, Finite groups, Second éd., Chelsea Publishing Co., New York, 1980. MR 81b:20002

[Hasse 1930] H. Hasse, "Neue Begründung und Verallgemeinerung der Theorie der Normenrestsymbols”, J. Reine Angew. Math. 162 (1930), 134-144.

[Kaplan 1976] P. Kaplan, "Sur le 2-groupe des classes d'idéaux des corps quadratiques", J. Reine Angew. Math. 283/284 (1976), 313-363. MR 53 \#8009

[Kisilevsky 1976] H. Kisilevsky, "Number fields with class number congruent to 4 mod 8 and Hilbert's theorem 94", J. Number Theory 8:3 (1976), 271-279. MR 54 \#5188 Zbl 0334.12019

[Kubota 1956] T. Kubota, "Über den bizyklischen biquadratischen Zahlkörper”, Nagoya Math. J. 10 (1956), 65-85. MR 18,643e

[Kučera 1995] R. Kučera, "On the parity of the class number of a biquadratic field", J. Number Theory 52:1 (1995), 43-52. MR 96e:11139

[Terada 1971] F. Terada, "A principal ideal theorem in the genus field", Tôhoku Math. J. (2) 23 (1971), 697-718. MR 46 \#5285 Zbl 0243.12003

[Wada 1966] H. Wada, "On the class number and the unit group of certain algebraic number fields", J. Fac. Sci. Univ. Tokyo Sect. I 13 (1966), 201-209 (1966). MR 35 \#5414

Received October 15, 2002.

\author{
ABDELMALEK AZIZI \\ DÉPARTEMENT DE MATHÉMATIQUES \\ FACULTÉ DES SCIENCES \\ UNIVERSITÉ MOHAMMED 1 \\ OUJDA \\ MAROC \\ azizi@sciences.univ-oujda.ac.ma \\ Ali Mouhib \\ DÉPARTEMENT DE MATHÉMATIQUeS \\ FACULTÉ DES SCIENCES \\ UNIVERSITÉ MOHAMMED 1 \\ OUJDA \\ MAROC
}

